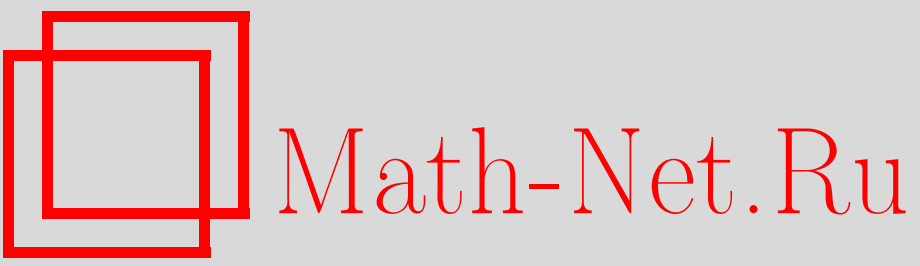

С. С. Акбаров, Стереотипные локально выпуклые пространства, Изв. РАН. Сер. матем., 2000, том 64, выпуск 4, 3-46

DOI: https://doi.org/10.4213/im294

Использование Общероссийского математического портала Math-Net.Ru подразумевает, что вы прочитали и согласны с пользовательским соглашением

http://www.mathnet.ru/rus/agreement

Параметры загрузки:

IP: 3.95 .254 .165

26 апреля 2023 г., 08:02:43 
УДК 519

\title{
С. С. Акбаров
}

\section{Стереотипные локально выпуклые пространства}

\author{
Приводятся доказательства некоторых анонсировавшихся ранее результатов \\ по теории стереотипных (рефлексивных по Понтрягину) локально выпуклых про- \\ странств, имеющих важные приложения в топологической алгебре и функцио- \\ нальном анализе. \\ Библиографояя: 18 наименований.
}

Теоремы двойственности Понтрягина-ван Кампена, доказанные в 30-х гг. для локально компактных абелевых групп [1], [2], были, как известно, обобщены потом на довольно широкий класс топологических абелевых групп (см., например, [3]), и, в частности, в 1952 г. М. Ф. Смит [4] было замечено, что всякое банахово пространство является рефлексивной по Понтрягину группой. Более точно этот результат формулируется следующим эквивалентным образом: всякое банахово пространство $X$ над $\mathbb{C}($ или $\mathbb{R})$ удовлетворяет тождеству

$$
\left(X^{\star}\right)^{\star} \cong X
$$

в котором каждая звездочка * обозначает сопряженное пространство линейных непрерывных функиионалов со значениями в $\mathbb{C}($ или $\mathbb{R}$ соответственно), наделенное топологией равномерной сходимости на вполне ограниченных множествах.

Пространства, выделяемые этим условием рефлексивности, изучались затем в 60-70-х гг. несколькими авторами, работавшими независимо друг от друга: Б. С. Брудовский [5], В. С. Уотерхаус [6] и К. Браунер [7] сделали первые наблюдения в этой области и, в частности, отметили, что этот класс весьма широк, потому что включает все квазиполные бочечные пространства. После этих публикаций, однако, тема была надолго забыта и появилась вновь лишш недавно в работах автора [8]-[10], где анонсировались некоторые приложения категории рефлексивных по Понтрягину локально выпуклых пространств (названных в [8] стереотипнылми) в функциональном анализе и теории гомологий топологических алгебр.

В настояшей работе мы приводим доказательства некоторых результатов работы [8]. Именно, мы описьваем конструкции псевдопополнения $X^{\nabla}$ и псевдонасьшения $X^{\Delta 1}$, доказываем анонсировавшиеся в [8] свойства категории Ste стереотипных пространств и приводим ряд важных технических результатов, которые автор надеется использовать в последуюших работах при доказательстве других фактов, анонсировавшихся в [8]-[10].

\footnotetext{
${ }^{1}$ Терминология и обозначения этой работы отличается от употреблявшихся в [8], [9]. В частности, $X^{\nabla}={ }^{\#} X, X^{\triangle}={ }_{b} X, X^{\star}=X^{\prime}$.
} 


\section{§ 0. Предварительные сведения}

0.1. Функционал Минковского и локально выпуклые топологии на векторном пространстве. Функиионалом Минковского множества В в векторном пространстве $X$ называется отображение $p: X \rightarrow \mathbb{R}_{+}$, действующее по формуле

$$
p(x)=\inf \{\lambda \geqslant 0: x \in \lambda B\}
$$

Функционал Минковского $p$ тогда и только тогда будет полунормой на $X$, когда $B$ выпукло, уравновешено и поглошает $X$.

Лемма 0.1. Пусть $р$ - функционал Минковского выпуклого уравновешенного поглощающего множества $B$ в векторном пространстве $X$ над $\mathbb{C}$. Тогда следующие условия әквивалентны:

(i) $B=\{x \in X: p(x) \leqslant 1\}$;

(ii) В оставляет замкнутый след на каждом одномерном подпространстве в $X:$ для всякого $x \in X$ мнохсество $\{\alpha \in \mathbb{C}: \alpha x \in B\}$ замкнуто в $\mathbb{C}$.

ДоказАтельство. Действительно, $a \in B$ означает, что $1 \in\{\lambda \geqslant 0: a \in \lambda B\}$, поэтому $p(x)=\inf \{\lambda \geqslant 0: x \in \lambda B\} \leqslant 1$. Наоборот, если $a \notin B$, то $1 \notin\{\lambda \geqslant 0: a \in$ $\lambda B\}$. Если выполнено (ii), то множество $\{\alpha \in \mathbb{C}: \alpha a \in B\}$ есть замкнутый круг в $\mathbb{C}$ с центром в нуле, поэтому $\sup \{\alpha \in \mathbb{C}: \alpha a \in B\}<1$. Отсюда $p(x)=\inf \{\lambda>0: x \in$ $\lambda B\}=\frac{1}{\sup \{\alpha>0: \alpha a \in B\}}>1$. Мы доказали импликацию (ii) $\Rightarrow(\mathrm{i})$. Обратная импликация очевидна.

Лемма 0.2. Система $\mathscr{V}$ выпуклых уравновешенных множеств в векторном пространстве $X$ над $\mathbb{C}$ тогда и только тогда является локальной базой некоторой отделимой локально выпуклой топологии $\tau$ на $X$, когда выполняются следующие условия:

(i) все множества $B \in \mathscr{V}$ поглощают $X: \bigcup_{\lambda \in \mathbb{C}} \lambda B=X$;

(ii) $\forall B \in \mathscr{V}, \quad \forall \lambda \in \mathbb{C} \backslash\{0\} \quad \exists C \in \mathscr{V}: C \subseteq \lambda B$;

(iii) $\forall B, C \in \mathscr{V} \quad \exists D \in \mathscr{V}: D \subseteq B \cap C$;

(iv) $\bigcap_{B \in \mathcal{V}} B=\{0\}$.

При этом:

(а) множество $B \in \mathscr{V}$ будет замкнуто относительно $\tau$ тогда и только тогда, когда оно оставляет замкнутый следна каждом одномерном подпространстве в $X:$ для всякого $x \in X$ множество $\{\alpha \in \mathbb{C}: \alpha x \in B\}$ замкнуто в $\mathbb{C} ;$

(b) система $\mathscr{V}$ будет совпадать с системой $\mathscr{Q} \mathscr{U}\left(X_{\tau}\right)$ всех выпуклых уравновешенных окрестностей нуля в $X$ (относительно $\tau$ ) тогда и только тогда, когда для выпуклого уравновешенного множества $M \subseteq X$ условие $\exists B \in \mathscr{V}: B \subseteq M$, автоматически влечет за собой условие $M \in \mathcal{V}$.

ДокАЗАТЕЛЬСтво. Свойство (i) означает, что функционалы Минковского (0.1) множеств $B \in \mathscr{V}$ являются полунормами на $X$, свойства (ii) и (iii) - что топология $\tau$, порожденная этими полунормами, имеет систему $\mathscr{V}$ своей локальной базой, свойство (iv) - что эта топология отделима. Свойство (a) следует из леммы 0.1, а свойство (b) очевидно. 
Следствие 0.3. Если выпуклая уравновешенная окрестность нуля $U$ в локально выпуклом пространстве $X$ оставляет замкнутый след на одномерных подпространствах в $X$, то $U$ - замкнутая окрестность нуля.

0.2. Вполне ограниченные, емкие и массивные в нуле множества. Если $X$ - локально выпуклое пространство (ЛВП) над $\mathbb{C}$, то условимся символом $\mathscr{U}(X)$ обозначать систему всех окрестностей нуля в $X$, а символом $\mathscr{K}(X)$ - систему всех компактов в $X$.

Напомним, что множество $S$ в ЛВП $X$ называется вполне ограниченным или $п р е д-$ компактныц, если для всякой окрестности нуля $U$ в $X$ найдется конечное множество $A$ такое, что $S \subseteq U+A$ [14]. Это равносильно тому, что $S$ вполне ограничено в смысле индуцированной из $X$ равномерной структуры [12] (т.е. A можно въбирать лежащим в $S)$.

Система всех вполне ограниченных множеств в $X$ обозначается $\mathscr{S}(X)$. Поскольку всякий компакт является вполне ограниченным множеством, справедливо вложение

$$
\mathscr{K}(X) \subseteq \mathscr{S}(X) .
$$

Предложение 0.4. Для множества $S$ в ЛВП Х следующие условия эквивалентны:

(i) $S$ вполне ограничено в $X$;

(ii) $\forall U \in \mathscr{U}(X) \quad \exists T \in \mathscr{S}(X): S \subseteq U+T$;

(iii) $\forall U \in \mathscr{U}(X) \quad \exists K \in \mathscr{K}(X): S \subseteq U+K$.

ОПредЕЛЕниЕ. Множество $D \subseteq X$ мы называем емким по отношению $\propto$ множеству $S \subseteq X$, если для всякого скаляра $\varepsilon \neq 0$ найдется конечное множество $A \subseteq X$ такое, что $S \subseteq \varepsilon D+A$. Полезно заметить, что если $D$ выпукло, то $A$ можно выбирать лежащим в $S$ (и при этом получится эквивалентное условие на $D$ ).

Лемма 0.5. Пересечение $C \cap D$ любых двух выпуклых уравновешенных множеств $C$ и $D$, емких по отношению $к S$, является выпуклым уравновешенныцм множеством, емким по отношению $\kappa S$.

ДокАЗАТЕЛЬСтво. Нужно проверить емкость $C \cap D$ по отношению к $S$. Пусть $\varepsilon \neq 0$. Выберем конечное множество $A \subseteq S$ так, чтобы $S \subseteq \frac{\varepsilon}{2} C+A$. Каждому $a \in A$ поставим в соответствие множество $S_{a}=S \cap\left(\frac{\varepsilon}{2} C+a\right)$. Поскольку $D$ выпукло и является емким по отношению к $S_{a}$, можно подобрать конечное $B_{a} \subseteq S_{a}$ так, чтобы $S_{a} \subseteq \varepsilon D+B_{a}$. Таким образом, мы получим конечное семейство конечных множеств $\left\{B_{a}, a \in A\right\}$. Положим $B=\bigcup_{a \in A} B_{a}$ и покажем, что

$$
S \subseteq \varepsilon(C \cap D)+B .
$$

Пусть $x \in S$. Тогда сушествует $a \in A$ такое, что $x \in S_{a} \subseteq \frac{\varepsilon}{2} C+a$, откуда

$$
x-a \in \frac{\varepsilon}{2} C .
$$

Поскольку, далее, $x \in S_{a} \subseteq \varepsilon D+B_{a}$, найдется $b \in B_{a} \subseteq B$ такое, что $x \in D+b$, т.е.

$$
x-b \in \varepsilon D .
$$


Наконец, поскольку $b \in B_{a} \subseteq S_{a} \subseteq \frac{\varepsilon}{2} C+a$, имеем

$$
b-a \in \frac{\varepsilon}{2} C .
$$

Множество $\varepsilon C$ выпукло и уравновешено, поэтому из $(0.3)$ и $(0.5)$ следует

$$
x-b=(x-a)-(b-a) \in \frac{\varepsilon}{2} C-\frac{\varepsilon}{2} C=\varepsilon C .
$$

Вместе с (0.4) это дает $x-b \in \varepsilon C \cap \varepsilon D=\varepsilon(C \cap D)$. Таким образом, имеем $x \in \varepsilon(C \cap$ $D)+b \subseteq \varepsilon(C \cap D)+B$, и мы доказали $(0.2)$.

ОПреДЕЛЕНИЕ. Множество $D$ в ЛВП $X$ мы называем емким, если оно является емким по отношению к любому вполне ограниченному множеству $S \subseteq X$. Это равносильно тому, что для любого $S \in \mathscr{S}(X)$ сушествует конечное $A \subseteq X$ такое, что $S \subseteq D+A$. Система всех емких множеств в $X$ обозначается $\mathscr{D}(X)$. Очевидно, что всякая окрестность нуля является емким множеством, поэтому справедливо включение

$$
\mathscr{U}(X) \subseteq \mathscr{D}(X)
$$

Лемма 0.6. Всякое выпуклое уравновешенное емкое множество $D$ в $X$ является поглощающим: $\bigcup_{\lambda \in \mathbb{C}} \lambda D=X$. Поэтому, в частности, всякое замкнутое выпуклое уравновешенное емкое мнохсество $D$ в $X$ является бочкой.

ДокАЗАтЕльство. Зафиксируем $x \in X$ и рассмотрим отрезок $[0, x]=\{\lambda x ; 0 \leqslant$ $\lambda \leqslant 1\}$. Поскольку он является компактом, найдется конечное множество $A \subseteq[0, x]$ такое, что $[0, x] \subseteq D+A$, т.е. отрезок $[0, x]$ покрывается конечной системой своих подынтервалов $I_{a}=[0, x] \cap(D+a), a \in A$. Ясно, что хотя бы один из этих интервалов должен быть невырож ден: $\exists a \in A: I_{a} \neq\{a\}$. Тогда $I_{a}$ содержит точку вида $\alpha x+a$, где $\alpha \in \mathbb{R} \backslash\{0\}$. Таким образом, $\alpha x+a \in I_{a} \subseteq D+a$, откуда $\alpha x \in D$ и, значит, $x \in \frac{1}{\alpha} D$.

ОПРЕДЕЛЕНИЕ. Множество $M$ в ЛВП $X$ мы называем массивным в нуле, если для любого вполне ограниченного множества $S$ в $X$ найдется окрестность нуля $U$ в $X$ такая, что $S \cap U \subseteq M$. Система всех массивных в нуле множеств в $X$ обозначается $\mathscr{M}(X)$. Очевидно, что всякая окрестность нуля является массивным в нуле множеством, а всякое массивное в нуле множество является емким. Поэтому

$$
\mathscr{U}(X) \subseteq \mathscr{M}(X) \subseteq \mathscr{D}(X)
$$

Теорема 0.7. Для замкнутого выпуклого уравновешенного множества $D$ в ЛВП Х следующие условия әквивалентны:

(i) $D$ является емким;

(ii) $D$ является массивным в нуле. 
ДокАЗАТЕльСтво. 1. Докажем импликацию (i) $\Rightarrow$ (ii), обратная импликация очевидна. Пусть $D \subseteq X$ - замкнутое, выпуклое, уравновешенное и емкое множество. Зафиксируем вполне ограниченное $S \subseteq X$ и покажем, что найдется конечное множество линейных непрерывных функционалов $F \subseteq X^{\prime}$ такое, что

$$
S \cap{ }^{\circ} F \subseteq D
$$

где ${ }^{\circ} F=\{x \in X: \forall f \in F|f(x)| \leqslant 1\}$ - обратная поляра множества $F$. Выберем конечное множество $A \subseteq X$ так, чтобы $2 S \subseteq D+A$, т.е.

$$
S \subseteq \frac{1}{2}(D+A)
$$

Положим $B=A \backslash D$ и заметим, что $\forall b \in B \quad 0 \notin \frac{1}{2}(D+b)$. Поэтому каждому $b \in B$ можно по теореме Хана-Банаха поставить в соответствие функционал $f_{b} \in X^{\prime}$, отделяющий $\frac{1}{2}(D+b)$ от нуля:

$$
\inf _{x \in \frac{1}{2}(D+b)}\left|f_{b}(x)\right| \geqslant 2
$$

Тогда

$$
\forall b \in B \quad \frac{1}{2}(D+b) \cap^{\circ} f_{b}=\varnothing
$$

Рассмотрим полученное конечное множество функционалов $F=\left\{f_{b} ; b \in B\right\}$. Для него, во-первых, оказывается

$$
\frac{1}{2}(D+B) \cap^{\circ} F=\bigcup_{b \in B}\left[\frac{1}{2}(D+b) \cap^{\circ} F\right] \subseteq \bigcup_{b \in B}\left[\frac{1}{2}(D+b) \cap^{\circ} f_{b}\right] \stackrel{(0.8)}{=} \varnothing
$$

и, во-вторых, поскольку $A \backslash B=A \backslash(A \backslash D)=A \cap D$, то

$$
\frac{1}{2}(D+(A \backslash B)) \cap{ }^{\circ} F \subseteq \frac{1}{2}(D+D) \cap{ }^{\circ} F=D \cap{ }^{\circ} F \subseteq D
$$

Формулы $(0.7),(0.9)$ и (0.10) вместе дают (0.6):

$S \cap{ }^{\circ} F \subseteq \frac{1}{2}(D+A) \cap{ }^{\circ} F \subseteq\left[\frac{1}{2}(D+B) \cap{ }^{\circ} F\right] \cup\left[\frac{1}{2}(D+(A \backslash B)) \cap^{\circ} F\right] \subseteq \varnothing \cup D=D$.

Итак, доказана формула (0.6), в которой ${ }^{\circ} F$ есть не что иное, как ( $X^{\prime}$-слабая) окрестность нуля $U \in \mathscr{U}(X): S \cap U \subseteq D$. Поскольку это верно для всякого $S \in \mathscr{S}(X)$, множество $D$ массивно в нуле. Теорема доказана. 
ОПРЕДЕЛЕНИЕ. Множество $B$ в $X$ мы называем предкомпактно-замкнуты.м, если оно оставляет замкнутый след $B \cap S$ на каждом замкнутом предкомпактном множестве $S \subseteq X$.

Пусть $\mathscr{Q}(X)$ обозначает систему всех выпуклых уравновешенных множеств в $X$, $\mathscr{P}(X)$ - систему всех предкомпактно-замкнутых выпуклых уравновешенных множеств в $X$, а $\mathscr{B}(X)$ - систему всех замкнутых выпуклых уравновешенных множеств в $X$. Положим

$$
\begin{aligned}
& \mathscr{B} \mathscr{D}(X)=\mathscr{B}(X) \cap \mathscr{D}(X), \quad \mathscr{P} \mathscr{D}(X)=\mathscr{P}(X) \cap \mathscr{D}(X), \quad \mathscr{Q} \mathscr{D}(X)=\mathscr{Q}(X) \cap \mathscr{D}(X), \\
& \mathscr{B} \mathscr{M}(X)=\mathscr{B}(X) \cap \mathscr{M}(X), \quad \mathscr{P} \mathscr{M}(X)=\mathscr{P}(X) \cap \mathscr{M}(X), \quad \mathscr{Q} \mathscr{M}(X)=\mathscr{Q}(X) \cap \mathscr{M}(X), \\
& \mathscr{B} \mathscr{U}(X)=\mathscr{B}(X) \cap \mathscr{U}(X), \quad \mathscr{P} \mathscr{U}(X)=\mathscr{P}(X) \cap \mathscr{U}(X), \quad \mathscr{Q} \mathscr{U}(X)=\mathscr{Q}(X) \cap \mathscr{U}(X), \\
& \mathscr{B} \mathscr{S}(X)=\mathscr{B}(X) \cap \mathscr{S}(X), \quad \mathscr{P} \mathscr{S}(X)=\mathscr{P}(X) \cap \mathscr{S}(X), \quad \mathscr{Q} \mathscr{S}(X)=\mathscr{Q}(X) \cap \mathscr{S}(X), \\
& \mathscr{B} \mathscr{K}(X)=\mathscr{B}(X) \cap \mathscr{K}(X), \quad \mathscr{P} \mathscr{K}(X)=\mathscr{P}(X) \cap \mathscr{K}(X), \quad \mathscr{Q} \mathscr{K}(X)=\mathscr{Q}(X) \cap \mathscr{K}(X) .
\end{aligned}
$$

Очевидно, эти системы связаны между собой соотношениями

$$
\begin{aligned}
& \mathscr{B} \mathscr{D}(X) \subseteq \mathscr{P} \mathscr{D}(X) \subseteq \mathscr{Q} \mathscr{D}(X) \\
& \| \quad U|\quad U| \mathscr{B} \mathscr{S}(X)=\mathscr{P} \mathscr{S}(X) \subseteq \mathscr{Q} \mathscr{S}(X) \\
& \mathscr{B} \mathscr{M}(X) \subseteq \mathscr{P} \mathscr{M}(X) \subseteq \mathscr{Q} \mathscr{M}(X) \quad \text { U UI U| } \\
& \text { UI Ul Ul } \mathscr{B} \mathscr{K}(X)=\mathscr{P} \mathscr{K}(X)=\mathscr{Q} \mathscr{K}(X) \\
& \mathscr{B} \mathscr{U}(X)=\mathscr{P} \mathscr{U}(X) \subseteq \mathscr{Q} \mathscr{U}(X)
\end{aligned}
$$

(равенство $\mathscr{B} \mathscr{U}=\mathscr{P} \mathscr{U}$ следует из следствия 0.3, а $\mathscr{B} \mathscr{D}=\mathscr{B} \mathscr{M}-$ из теоремы 0.7).

Предложение 0.8. Пусть $\mathscr{V}$ - какая-нибудь из систем множеств $\mathscr{B D}, \mathscr{P} \mathscr{D}$, $\mathscr{B} \mathscr{M}, \mathscr{P} \mathscr{M}, \mathscr{Q} \mathscr{M}$. Тогда:

(i) $\forall D \in \mathscr{V} \quad \bigcup_{\lambda \in \mathbb{C}} \lambda D=X$;

(ii) $\forall D \in \mathscr{V}, \quad \forall \lambda \in \mathbb{C} \backslash\{0\} \quad \lambda D \in \mathscr{V}$;

(iii) $\forall C, D \in \mathscr{V} \quad C \cap D \in \mathscr{V}$;

(iv) $\bigcap_{D \in \mathscr{V}} D=\{0\}$.

0.3. Дуальные системы и поляры. Напомним, что если на паре векторных пространств $(P, Q)$ определена невырожденная билинейная форма $\langle\cdot, \cdot\rangle: P \times Q \rightarrow \mathbb{C}$, то такая пара называется дуальной системой. Полярами множеств $G \subseteq P$ и $H \subseteq Q$ называются множества

$$
G^{\circ}=\{y \in Q: \forall x \in G|\langle x, y\rangle| \leqslant 1\}, \quad{ }^{\circ} H=\{x \in P: \forall y \in H|\langle x, y\rangle| \leqslant 1\} .
$$

Лемма 0.9. Пусть $\langle P, Q\rangle-$ дуальная система векторных пространств над $\mathbb{C}$, и пусть заданы множество $G \subseteq P$, выпуклое, уравновешенное и $Q$-слабо замкнутое в $P$, имножество $H \subseteq Q$, выпуклое, уравновешенное и $P$-слабо компактное в $Q$.

Тогда $\left(G \cap{ }^{\circ} H\right)^{\circ} \subseteq G^{\circ}+H$.

ДокАЗАТЕльСтво. Из включения $G^{\circ}+H \supseteq G^{\circ} \cup H$ следует $^{\circ}\left(G^{\circ}+H\right) \subseteq{ }^{\circ}\left(G^{\circ} \cup H\right)=$ ${ }^{\circ}\left(G^{\circ}\right) \cap{ }^{\circ} H=G \cap{ }^{\circ} H$. Множество $G^{\circ} P$-слабо замкнуто, а $H \quad P$-слабо компактно, поэтому $G^{\circ}+H P$-слабо замкнуто. Отсюда $G^{\circ}+H=\left[{ }^{\circ}\left(G^{\circ}+H\right)\right]^{\circ} \supseteq\left(G \cap{ }^{\circ} H\right)^{\circ}$. 


\section{§ 1. Функторы $\vee, \wedge, \nabla, \triangle, \nabla, \wedge$}

1.1. Функтор пополнения $\nabla$ и плотные вложения. ЛВП $X$ мы, как обычно, называем полнылм, если в нем всякая направленность Коши сходится. Как известно, всякое ЛВП обладает пополнением, т.е. ближайшим к нему снаружи полным пространством. Формально эту конструкцию можно описать следующим образом.

Теорема 1.1. Каждому ЛВП $Х$ можно поставить в соответствие морфизм $\boldsymbol{\nabla}_{X}: X \rightarrow X^{\boldsymbol{\nabla}}$ в некоторое полное ЛВП $X^{\boldsymbol{\nabla}}$ так, чтобы выполнялись условия:

(i) $X$ полно, тогда и только тогда, когда $\boldsymbol{\nabla}_{X}: X \rightarrow X^{\boldsymbol{\nabla}}-$ изоморфизм;

(ii) для любого морфизма ЛВП $\varphi: X \rightarrow Y$ найдется единственный морфизм ЛВП $\varphi^{\mathbf{\nabla}}: X^{\mathbf{\nabla}} \rightarrow Y^{\mathbf{\nabla}}$, замыкающий диаграмму

Из второго условия следует, что отображение $\varphi \mapsto \varphi^{\mathbf{\nabla}}$ является ковариантным функтором категории $\mathfrak{L} \mathfrak{C S}$ в себя: $(\psi \circ \varphi)^{\boldsymbol{\nabla}}=\psi^{\boldsymbol{\nabla}} \circ \varphi^{\boldsymbol{\nabla}}$. Заметим также, что если $\varphi: X \rightarrow Y$ - морфизм в полное ЛВП $Y$, то сушествует единственный морфизм $X^{\boldsymbol{\nabla}} \rightarrow Y$, замыкаюший диаграмму

Это, между прочим, означает, что пространство $X^{\boldsymbol{\nabla}}$ и морфизм $\boldsymbol{\nabla}_{X}: X \rightarrow X^{\boldsymbol{\nabla}}$ определены однозначно, с точностью до изоморфизма. Действительно, если бы морфизм $\boldsymbol{\nabla}$ можно было определить двумя разными способами:

$$
\boldsymbol{\nabla}_{X}: X \rightarrow X^{\mathbf{\nabla}}, \quad \tilde{\boldsymbol{\nabla}}_{X}: X \rightarrow \tilde{X}^{\boldsymbol{\nabla}},
$$

то возникли бы две коммутативные диаграммы 
(в которых каждый внутренний треугольник с пунктирной стороной - это маленькая диаграмма (1.2)). В силу единственности пунктирных стрелок морфизмы $\alpha$ и $\beta$ слева те же самые, что $\alpha$ и $\beta$ справа. Поэтому равенства

$$
\beta \circ \alpha=\mathbf{1}_{X} \mathbf{v}, \quad \alpha \circ \beta=\mathbf{1}_{\widetilde{X}^{\mathbf{v}}}
$$

означают, что $\alpha$ и $\beta$ - изоморфизмы.

Таким образом, конструкция $\boldsymbol{\nabla}_{X}: X \rightarrow X^{\boldsymbol{\nabla}}$ однозначна, и это оправдьвает следующее

ОПреДЕЛЕниЕ. Пространство $X^{\boldsymbol{\nabla}}$ назьвается пополнением, а морфизм $\boldsymbol{\nabla}_{X}$ : $X \rightarrow X^{\mathbf{\nabla}}$ - отображснием пополнения ЛВП $X$. Ковариантный функтор $\varphi \rightarrow \varphi^{\boldsymbol{\nabla}}$ категории $\mathfrak{L} \mathfrak{C S}$ в себя называется функтором пополнения.

Морфизм ЛВП $\sigma: X \rightarrow Y$ мы называем вложсением, если $\sigma$ инъективно и открыто (т.е. $\forall U \in \mathscr{U}(X) \exists V \in \mathscr{U}(Y): U \supseteq V \cap \sigma(X))$, и плотным вложсением, если вдобавок образ $\sigma(X)$ плотен в $Y$.

Очевидным примером плотного вложения является отображение пополнения $\boldsymbol{\nabla}_{X}: X \rightarrow$ $X^{\mathbf{\nabla}}$. Эта конструкция обладает естественным свойством универсальности в системе всех плотных вложений пространства $X$, потому что любое плотное вложение “лежит между $X$ и $X^{\mathbf{\nabla}}$.

Теорема 1.2. Морфизм ЛВП $\sigma: X \rightarrow Y$ тогда и только тогда является плотным вложсением, когда он вписъвается в (необходимо единственную) коммутативную диаграмму

в которой:

- $X^{\sigma}$ обозначает некоторое подпространство в пополнении $X^{\boldsymbol{\nabla}}$ пространства $X$, содерэсащее $X$;

- морфизмы $\alpha$ и $\beta$ изображают иепочку вложений $X \subseteq X^{\sigma} \subseteq X^{\mathbf{\nabla}}$;

- $Y \cong X^{\sigma}-$ некоторый изоморфизм ЛВП.

1.2. Функтор насышения $\triangle$ и точные наложения. Пусть $X$ - произвольное локально выпуклое пространство и $\tau$ обозначает его топологию. В силу предложения 0.8 и леммы 0.2 система $\mathscr{Q} \mathscr{M}(X)$ выпуклых уравновешенных массивных в нуле множеств в $X$ является локальной базой некоторой отделимой локально выпуклой топологии $\tau^{\boldsymbol{\Delta}}$ на $X$, мажорируюшей топологию $\tau\left(\tau \subseteq \tau^{\mathbf{\Delta}}\right)$. Обозначим через $X^{\mathbf{\Delta}}$ пространство $X$ с этой новой топологией $\tau^{\mathbf{\Lambda}}$. В силу очевидного для $\mathscr{Q} \mathscr{M}(X)$ условия (b) леммы 0.2 справедливо равенство

$$
\mathscr{Q} \mathscr{M}(X)=\mathscr{Q} \mathscr{U}\left(X^{\mathbf{\Lambda}}\right)
$$


Заметим, что топология $\tau^{\mathbf{\Delta}}$ на X имеет ту же самую систему вполне ограниченных множеств, что и $\tau$ :

$$
\mathscr{S}\left(X^{\mathbf{\Delta}}\right)=\mathscr{S}(X)
$$

причем на каждом вполне ограниченном множестве $S \subseteq X$ топологии $\tau$ и $\tau^{\mathbf{\Delta}}$ совпадают. Отсюда следуют равенства

$$
\mathscr{M}\left(X^{\mathbf{\Delta}}\right)=\mathscr{M}(X), \quad \mathscr{P}\left(X^{\mathbf{\Delta}}\right)=\mathscr{P}(X),
$$

которые вместе с (1.3) дают

$$
\begin{aligned}
& \mathscr{Q} \mathscr{M}\left(X^{\mathbf{\Delta}}\right)=\mathscr{Q}\left(X^{\mathbf{\Delta}}\right) \cap \mathscr{M}\left(X^{\mathbf{\Delta}}\right)=\mathscr{Q}(X) \cap \mathscr{M}(X)=\mathscr{Q} \mathscr{M}(X), \\
& \mathscr{P} \mathscr{M}\left(X^{\mathbf{\Delta}}\right)=\mathscr{P}\left(X^{\mathbf{\Delta}}\right) \cap \mathscr{M}\left(X^{\mathbf{\Delta}}\right)=\mathscr{P}(X) \cap \mathscr{M}(X)=\mathscr{P} \mathscr{M}(X) .
\end{aligned}
$$

Собирая вместе эти связи, мы получим две цепочки равенств:

$$
\begin{aligned}
& \mathscr{Q} \mathscr{M}(X)=\mathscr{Q} \mathscr{M}\left(X^{\mathbf{\Delta}}\right)=\mathscr{Q} \mathscr{U}\left(X^{\mathbf{\Delta}}\right) \\
& \mathscr{P} \mathscr{M}(X)=\mathscr{P} \mathscr{M}\left(X^{\mathbf{\Delta}}\right)=\mathscr{P} \mathscr{U}\left(X^{\mathbf{\Delta}}\right)=\mathscr{B} \mathscr{U}\left(X^{\mathbf{\Delta}}\right)
\end{aligned}
$$

(последнее равенство $\mathscr{P} \mathscr{U}=\mathscr{B} \mathscr{U}$ справедливо в силу следствия 0.3).

ОПРЕДЕЛЕНИЕ. ЛВП $X$ называем насыщеннылм, если в нем всякое выпуклое уравновешенное массивное в нуле множество $M$ является окрестностью нуля.

Это означает выполнение равенства $\mathscr{Q} \mathscr{M}(X)=\mathscr{Q} \mathscr{U}(X)$, т.е. в силу (1.4) совпадение топологий $\tau=\tau^{\boldsymbol{\Delta}}$. Можно заметить, что насышенность пространства $X$ равносильна тому, что в $X$ всякое предкомпактно-замкнутое выпуклое уравновешенное массивное в нуле множество $M$ является окресностью нуля, т.е. справедливо равенство

$$
\mathscr{P} \mathscr{M}(X)=\mathscr{P} \mathscr{U}(X)=\mathscr{B} \mathscr{U}(X)
$$

Действительно, поскольку в $X^{\boldsymbol{\Delta}}$, как в любом локально выпуклом пространстве, замкнутые выпуклые уравновешенные окрестности нуля образуют локальнуюбазу, мы получаем в силу (1.4), что $\mathscr{P} \mathscr{M}(X)$ конфинально сидит в $\mathscr{Q} \mathscr{M}(X)$, т.е. что всякое выпуклое уравновешенное массивное в нуле множество $M \subseteq X$ содержит некоторое предкомпакно-замкнутое выпуклое уравновешенное массивное в нуле множество $N \subseteq X$. Следовательно, если всякое множество $N \in \mathscr{P} \mathscr{M}(X)$ является окрестностью нуля в $X$, то и всякое множество $M \in \mathscr{Q} \mathscr{M}(X)$ должно быть окрестностью нуля в $X$.

Равенство $\mathscr{Q} \mathscr{M}(X)=\mathscr{Q} \mathscr{M}\left(X^{\mathbf{\Delta}}\right)$ означает, между прочим, что для всякого локально выпуклого пространства $X$ соотвествуюшее пространство $X^{\mathbf{\Delta}}$ является насышенным. Обозначив через $\boldsymbol{\Delta}_{X}: X^{\boldsymbol{\Delta}} \rightarrow X$ отображение, соответствуюшее вложению топологий $\tau^{\mathbf{\Perp}} \supseteq \tau$, мы получим конструкцию, двойственную в некотором смысле конструкции пополнения. 
Теорема 1.3. Каждому ЛВП $Х$ мохно поставить в соответствие морфизм $\boldsymbol{\Delta}_{X}: X^{\mathbf{\Delta}} \rightarrow X$ из некоторого насыщенного ЛВП $X^{\mathbf{\Delta}}$ так, чтобы выполнялись условия:

(i) $X$ насыщено тогда и только тогда, когда $\boldsymbol{\Delta}_{X}: X^{\mathbf{\Delta}} \rightarrow X-$ изоморфизм;

(ii) для любого морфизма ЛВП $\varphi: Y \rightarrow X$ найдется единственный морфизм ЛВП $\varphi^{\mathbf{\Lambda}}: Y^{\mathbf{\Delta}} \rightarrow X^{\mathbf{\Lambda}}$, замыкающий диаграмму

Понятно, что отображение $\varphi \mapsto \varphi^{\mathbf{\Delta}}$ есть ковариантный функтор категории $\mathfrak{L} \mathfrak{C}$ в себя: $(\psi \circ \varphi)^{\mathbf{\Lambda}}=\psi^{\mathbf{\Lambda}} \circ \varphi^{\mathbf{\Lambda}}$. Ясно также, что если $\varphi: Y \rightarrow X-$ морфизм из насышенного ЛВП $Y$, то существует единственный морфизм ЛВП $\varphi: Y \rightarrow X^{\boldsymbol{\Delta}}$, замыкающий диаграмму

Как и в случае с пополнением, это означает, в частности, что пространство $X^{\boldsymbol{\Delta}}$ и морфизм $\boldsymbol{\Delta}_{X}: X^{\boldsymbol{\Delta}} \rightarrow X$ определены однозначно с точностью до изоморфизма. Поэтому имеет смысл

ОПреДЕЛЕНИЕ. Пространство $X^{\boldsymbol{\Delta}}$ называется насыщением, а морфизм $\boldsymbol{\Delta}_{X}$ : $X^{\boldsymbol{\Delta}} \rightarrow X$ - отображсением насыщения пространства $X$. Ковариантньй функтор $\varphi \mapsto \varphi^{\mathbf{\Lambda}}$ категории $\mathfrak{L} \mathfrak{C} \mathfrak{S}$ в себя называется функтором насыщения.

Морфизм ЛВП $\pi: Y \rightarrow X$ мы называем

- наложением, если всякое вполне ограниченное множество $S \subseteq X$ содержится в образе $\pi(T)$ некоторого вполне ограниченного множества $T \subseteq Y$ :

$$
\forall S \in \mathscr{S}(X) \quad \exists T \in \mathscr{S}(Y): \quad \pi(T) \supseteq S ;
$$

- точным наложением, если вдобавок для всякого вполне ограниченного множества $T \subseteq Y$ ограничение $\left.\pi\right|_{T}: T \rightarrow \pi(T)$ является гомеоморфизмом топологических пространств.

Заметим, что наложение всегда является сюръекцией, а точное наложение - биекцией. Поэтому если пространства $Y$ и $X$ связаны точным наложением $\pi: Y \rightarrow X$, то $Y$ можно рассматривать как новую, более тонкую топологизацию пространства $X$, сохраняющую вполне ограниченные множества и топологию на вполне ограниченных множествах. Именно такой топологизацией является насышение $X^{\boldsymbol{\Delta}}$ пространства $X$, конструктивно описанное нами в начале этого пункта. Поскольку насышение единственно, мы получаем, что отображение насышения $\boldsymbol{\Delta}_{X}: X^{\boldsymbol{\Delta}} \rightarrow X$ всякого ЛВП $X$ является точным наложением. Более того, из определения топологии $X^{\boldsymbol{\Delta}}$ следует, что всякое, вообше точное, наложение естественно "вписывается" в отображение насышения. 
Теорема 1.4. Морфизм ЛВП $\pi: Y \rightarrow X$ тогда и только тогда будет точныцм наложением, когда он вписывается в (необходимо единственную) коммутативную диаграмму

в которой:

- $X_{\pi}$ обозначает пространство $X$ с новой топологией $\pi$, лежащей между топологиями $X$ и $X^{\mathbf{4}}$;

- морфизмы $\beta$ и а изображают последовательное ослабление топологии от $X^{\mathbf{\Lambda}} \kappa X_{\pi}$ u om $X_{\pi} \kappa X$

$-Y \cong X_{\pi}-$ некоторьй изоморфизм ЛВП.

1.3. Псевдополнота и псевдопополнение (функтор $\nabla$ ). ЛВП $X$ мы называем псевдополныц, если в нем всякая вполне ограниченная направленность Коши сходится. Это равносильно тому, что всякое замкнутое вполне ограниченное множество $S$ в $X$ является компактом. С обычной полнотой и квазиполнотой это условие связано импликациями:

$$
\text { полнота } \Longrightarrow \text { квазиполнота } \Longrightarrow \text { псевдополнота. }
$$

Поэтому всякое (полное и всякое) квазиполное пространство псевдополно. В метризуемом случае эти понятия эквивалентны.

Оказывается, что, как и в случае с полнотой, всякое ЛВП $X$ обладает псевдопополнением, т.е. ближайшим к нему "снаружи” псевдополным пространством. Формально эту конструкцию описывает следуюшая

Теорема 1.5. Каждому ЛВП Х можно поставить в соответствие морфизм $\nabla_{X}: X \rightarrow X^{\nabla}$ в некоторое псевдополное ЛВП $X^{\nabla}$ так, чтобы выполнялись условия:

(i) $X$ псевдополно тогда и только тогда, когда $\nabla_{X}: X \rightarrow X^{\nabla}$ - изоморфизм;

(ii) для любого морфизма ЛВП $\varphi: X \rightarrow Y$ найдется единственный морфизм ЛВП $\varphi^{\nabla}: X^{\nabla} \rightarrow Y^{\nabla}$, замыкающий диаграмму

Из второго условия следует, что отображение $\varphi \mapsto \varphi^{\nabla}$ является ковариантным функтором категории $\mathfrak{L C S}$ в себя: $(\psi \circ \varphi)^{\nabla}=\psi^{\nabla} \circ \varphi^{\nabla}$. Заметим также, что если $\varphi: X \rightarrow Y$ 
- морфизм в полное ЛВП $Y$, то сушествует единственный морфизм $X^{\nabla} \rightarrow Y$, замыкающий диаграмму

Это, в свою очередь, означает, что пространство $X^{\nabla}$ и морфизм $\nabla_{X}: X \rightarrow X^{\nabla}$ определены однозначно с точностью до изоморфизма. Таким образом, конструкция $\nabla_{X}: X \rightarrow$ $X^{\nabla}$ однозначна, и это оправдывает следуюшее

ОПРЕДЕлЕнИЕ. Пространство $X^{\nabla}$ называется псевдопополнением, а морфизм $\nabla_{X}: X \rightarrow$ $X^{\nabla}$ - отображсением псевдопополнения ЛВП $X$. Ковариантный функтор $\varphi \rightarrow \varphi^{\nabla}$ категории $\mathfrak{L} \mathfrak{C S}$ в себя называется функтором псевдопополнения.

Теорема 1.6. Для всякого ЛВП $X$ отображсение псевдопополнения $\nabla_{X}$ : $X \rightarrow X^{\nabla}$ является плотным вложением.

Доказательство теорем 1.5 и 1.6 мы проведем в три этапа.

(а) Функтор $\vee$. Каждому ЛВП $X$ поставим в соответствие подпространство $X^{\vee}$ в пополнении $X^{\mathbf{v}}$, состоящее из пределов всевозможных вполне ограниченных направленностей Коши из $X$ :

$$
x \in X^{\vee} \Longleftrightarrow x \in X^{\nabla} \quad \& \quad \exists\left\{x_{i}\right\} \in \mathscr{S}(X): \quad x_{i} \stackrel{X^{\nabla}}{\longrightarrow} x \quad(i \rightarrow \infty) .
$$

Пространство $X^{\vee}$ наделяется топологией, индуцированной из $X^{\vee}$. Естественная инъекция $X$ в $X^{\vee}$ обозначается $\vee_{X}: X \rightarrow X^{\vee}$.

Лемма 1.7. Отображсение $\vee_{X}: X \rightarrow X^{\vee}$ является плотным вложсением,

(i) $X$ псевдополно тогда и только тогда, когда $\vee_{X}: X \rightarrow X^{\vee}-$ изоморфизм;

(ii) для любого морфизма ЛВП $\varphi: X \rightarrow Y$ найдется единственный морфизм ЛВП $\varphi^{\vee}: X^{\vee} \rightarrow Y^{\vee}$, замыкающий диаграмму

Отсюда следует, что если $\varphi: X \rightarrow Y$ - морфизм в псевдополное ЛВП $Y$, то сушествует единственный морфизм $X^{\vee} \rightarrow Y$, замыкающий диаграмму 
(b) Инъективный ряд. Инвективнылм рядом локально выпуклого пространства $X$ мы называем трансфинитную последовательность локально выпуклых пространств $\left\{X^{\omega} ; \omega \in\right.$ ORD $\}$ (занумерованных ординальными числами - см. [13]) с системой морфизмов ЛВП $\left\{\sigma_{\iota}^{\varkappa}: X^{\iota} \rightarrow X^{\varkappa}, \iota<\varkappa \in \mathrm{ORD}\right\}$, удовлетворяюшей следуюшим условиям:

(i) $X^{0}=X$;

(ii) для любых трех ординалов $\iota<\varkappa<\lambda$ коммутативна диаграмма

(iii) если $\lambda$ - изолированный ординал, т.е. существует ординал $\varkappa$ такой, что

$$
\lambda=\varkappa+1=\min \{\omega \in \mathrm{ORD}: \varkappa<\omega\},
$$

то существует изоморфизм $X^{\lambda}=X^{\varkappa+1} \cong\left(X^{\varkappa}\right)^{\vee}$ такой, что

(iv) если $\lambda$ - предельный ординал, т.е. (1.12) не выполняется ни для какого $\varkappa$, то существует изоморфизм между $X^{\lambda}$ и локально выпуклым индуктивным пределом системы $\left\{X^{\iota} ; \iota<\lambda, \iota \rightarrow \lambda\right\}$ такой, что для всякого $\varkappa<\lambda$ коммутативна диаграмма

в которой $\rho_{\varkappa}-$ естественная инъекция элемента $X^{\varkappa}$ индуктивной системы $\left\{X^{\iota} ; \iota<\lambda\right\}$ в соответствующий индуктивный предел.

Трансфинитной индукцией доказывается

Лемма 1.8. Всякое ЛВП Х обладает инвективным рядом.

Из леммы 1.8 и диаграмм (1.13) и (1.14) следует

Лемма 1.9. Все морфизмы $\sigma_{\iota}^{\varkappa}: X^{\iota} \rightarrow X^{\varkappa}$ инбективного ряда произвольного данного ЛВП $Х$ являются плотными вложениями.

Из нее в свою очередь вытекает 
Лемма 1.10. Инбективный ряд данного ЛВП $X$ определяется однозначно $с$ точностью до изоморфизма.

Это означает, что если $\left\{X^{\omega}, \sigma_{\iota}^{\varkappa}\right\}$ и $\left\{\widetilde{X}^{\omega}, \tilde{\sigma}_{\iota}^{\varkappa}\right\}$ - два инъективных ряда для $X$, то сушествует система изоморфизмов $X^{\omega}=\widetilde{X}^{\omega}$ такая, что коммутативна диаграмма

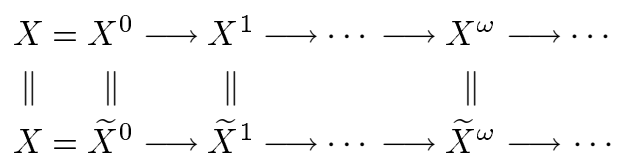

Лемма 1.11. Инбективнъй ряд $\left\{X^{\omega}, \sigma_{\iota}^{\varkappa}\right\}$ всякого ЛВП $Х$ стабилизируется: существует ординал $\mu$ такой, что

$$
\forall \omega>\mu \quad X^{\omega}=X^{\mu}
$$

(т.е. $\sigma_{\mu}^{\omega}: X^{\mu} \rightarrow X^{\omega}$ является изоморфизмом при $\left.\omega>\mu\right)$.

ДоКАЗАТЕЛЬСТво. Из леммы 1.9 и теоремы 1.2 следует, что все пространства $X^{\omega}$ можно считать вложенными в пополнение $X^{\boldsymbol{\nabla}}$ пространства $X$ :

$$
X=X^{0} \subseteq X^{1} \subseteq \cdots \subseteq X^{\omega} \subseteq \cdots \subseteq X^{\mathbf{\nabla}}
$$

Если предположить, что последовательность $X^{\omega}$ не стабилизируется, т.е.

$$
\forall \omega \in \text { ORD } \exists x^{\omega} \in X^{\omega+1} \backslash X^{\omega}
$$

то класс элементов $\left\{x^{\omega} ; \omega \in \mathrm{ORD}\right\}$ должен иметь “неограниченную мощность", т.е. не может быть множеством по Гёделю-Бернайсу [13]. Но с другой стороны, все $x^{\omega}$ лежат во множестве $X^{\boldsymbol{\nabla}}$ и, значит, класс $\left\{x^{\omega} ; \omega \in \mathrm{ORD}\right\}$ является множеством. Полученное противоречие означает, что

$$
\exists \mu \in \mathrm{ORD}: \quad X^{\mu+1}=X^{\mu},
$$

а отсюда уже следует (1.16).

Лемма 1.12. Пусть $\left\{X^{\omega} ; \omega \in \mathrm{ORD}\right\}-$ - илгективный ряд для ЛВП $X,\left\{Y^{\omega} ; \omega \in\right.$ ORD $\}$ - ингективныи ряд для ЛВП $Y$. Тогда для любого морфизма $\varphi: X \rightarrow$ $Y$ найдется единственная последовательность морфизмов $\left\{\varphi^{\omega}: X^{\omega} \rightarrow Y^{\omega} ; \omega \in\right.$ ORD\} такая, что коммутативна следующая диаграмма:

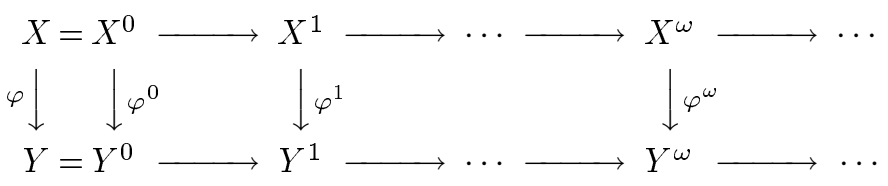


ДокАЗАТЕльство. Вначале полагаем $\varphi^{0}=\varphi$. Затем, если для некоторого ординала $\lambda$ все $\left\{\varphi^{\varkappa} ; \varkappa<\lambda\right\}$ уже построены, то рассматриваются два случая:

1) если $\lambda$ - изолированный ординал, т.е. выполняется (1.12), то с помошью леммы 1.7 полагаем

$$
\varphi^{\lambda}=\varphi^{\varkappa+1}=\left(\varphi^{\varkappa}\right)^{\vee}
$$

2) если же $\lambda$-предельный ординал, то, считая $X^{\omega}$ и $Y^{\omega}$ реализованными как системы подпространств в пополнениях $X^{\boldsymbol{\nabla}}$ и $Y^{\boldsymbol{\nabla}}$, мы сможем переписать диаграмму (1.14) для $X^{\omega}$ и $Y^{\omega}$ в виде равенств

$$
X^{\lambda}=\bigcup_{\iota<\lambda} X^{\iota}, \quad Y^{\lambda}=\bigcup_{\iota<\lambda} Y^{\iota}
$$

поэтому $\varphi^{\lambda}$ будет однозначно определяться своими ограничениями $\left.\varphi^{\lambda}\right|_{X^{\iota}}=\varphi^{\iota}$ : $X^{\iota} \rightarrow Y^{\iota}(\iota<\lambda)$; причем непрерывность $\varphi^{\lambda}$ будет следовать из определения индуктивного предела, а единственность - из леммы 1.9.

Лемма 1.12 доказана.

(c) Конструкция $X^{\nabla}$. Рассмотрим произвольное ЛВП $X$. Его инъективный ряд $\left\{X^{\omega} ; \omega \in\right.$ ORD $\}$ по лемме 1.11 стабилизируется. Пусть $\mu$ - ординал, на котором происходит эта стабилизация. Положив

$$
X^{\nabla}=X^{\mu}, \quad \nabla_{X}=\sigma_{0}^{\mu},
$$

мы получим, что, во-первых, пространство $X^{\nabla}$ псевдополно, потому что

$$
\left(X^{\nabla}\right)^{\vee}=\left(X^{\mu}\right)^{\vee}=X^{\mu+1}=X^{\mu}=X^{\nabla},
$$

во-вторых, если $X$ псевдополно, то по лемме $1.7\left(\sigma_{0}^{1}: X^{0} \rightarrow X^{1}\right)=\left(\vee_{X}: X \rightarrow X^{\vee}\right)-$ изоморфизм, откуда по индукции следует, что в инъективном ряде $\left\{X^{\omega}, \sigma_{\iota}^{\varkappa}\right\}$ все морфизмы $\sigma_{\iota}^{\varkappa}$ являются изоморфизмами. В частности, $\vee_{X}=\sigma_{0}^{\mu}$ тоже будет изоморфизмом. Наконец, в-третьих, сушествование и единственность морфизма $\varphi^{\nabla}$ в диаграмме (1.7) следует из леммы 1.12 .

Таким образом, доказана теорема 1.5. Теорема 1.6 следует из леммы 1.9.

1.4. Псевдонасыщенность и псевдонасышение (функтор $\triangle$ ). ЛВП $X$ мы называем псевдонасыщенным. если в нем всякое замкнутое выпуклое уравновешенное емкое множество $D$ является окрестностью нуля.

ПримеР 1.13. Всякое бочечное пространство псевдонасьшено в силу леммы 0.6 .

ПримеР 1.14. Всякое метризуемое (необязательно полное) ЛВП псевдонасыщено. Это вытекает из следуюшей леммы.

Лемма 1.15. Пусть $X$ - метризуемое ЛВП. Для всякого вполне ограниченного множества в его пополнении $T \subseteq X^{\mathbf{V}}$ найдется вполне ограниченное множество $S \subseteq X$, плотное в $T: T \subseteq \bar{S}$.

Замечательно, что сушествует стандартная конструкция, двойственная в определенном смысле конструкции псевдопополнения (см. ниже результаты $\S 3$ ), позволяюшее каждому ЛВП $X$ поставить в соответствие ближайшее к нему "изнутри" псевдонасышенное ЛВП $X^{\Delta}$. 
Теорема 1.16. Каждому ЛВП $Х$ можсно поставить в соответствие морфизм $\triangle_{X}: X^{\Delta} \rightarrow X$ из некоторого псевдонасыщенного ЛВП $X^{\Delta}$ так, чтобь выполнялись условия:

(i) $X$ псевдонасыщено тогда и только тогда, когда $\triangle_{X}: X^{\Delta} \rightarrow X-$ изоморфизм;

(ii) для любого морфизма ЛВП $\varphi: Y \rightarrow X$ найдется единственный морфизм ЛВП $\varphi^{\Delta}: Y^{\Delta} \rightarrow X^{\Delta}$, замыкающий диаграмму

Понятно, что отображение $\varphi \mapsto \varphi^{\Delta}$ есть ковариантный функтор категории $\mathfrak{L} \mathfrak{C}$ в себя: $(\psi \circ \varphi)^{\Delta}=\psi^{\Delta} \circ \varphi^{\Delta}$. Ясно также, что если $\varphi: Y \rightarrow X-$ морфизм из псевдонасыщенного ЛВП $Y$, то существует единственный морфизм ЛВП $\varphi: Y \rightarrow X^{\Delta}$, замыкающий диаграмму

Как и в случае с псевдопополнением, это означает, в частности, что пространство $X^{\triangle}$ и морфизм $\triangle_{X}: X^{\Delta} \rightarrow X$ определены однозначно с точностью до изоморфизма. Поэтому имеет смысл

ОПрЕДЕЛЕНИЕ. Пространство $X^{\triangle}$ назьвается псевдонасыщением, а морфизм $\triangle_{X}: X^{\triangle} \rightarrow$ $X$-отображсением псевдонасыщения пространства $X$. Ковариантньй функтор $\varphi \mapsto$ $\varphi^{\Delta}$ категории $\mathfrak{L} \mathfrak{C S}$ в себя назьвается функтором псевдонасыщения.

Теорема 1.17. Для всякого ЛВП $X$ отображсение псевдонасыщения $\triangle_{X}$ : $X^{\Delta} \rightarrow X$ является точным наложсением.

Таким образом, $X^{\Delta}$ можно рассматривать как новую, более тонкую топологизацию $X$, при которой сохраняется система вполне ограниченных множеств

$$
T \in \mathscr{S}\left(X^{\Delta}\right) \Longleftrightarrow \Delta_{X}(T) \in \mathscr{S}(X),
$$

причем топология на каждом вполне ограниченном множестве также не меняется.

Теоремы 1.16 и 1.17 доказываются по той же схеме, что и теоремы 1.5 и 1.6, и внешняя разница состоит лишь в обращении стрелок. Как и в п. 1.3, доказательство проводится в три этапа.

(a) Функтор $\wedge$. Для каждого ЛВП $X$ обозначим через $X^{\wedge}$ новую топологизацию пространства $X$, в которой локальной базой служат всевозможные замкнутые выпуклые уравновешенные емкие множества из $X$ :

$$
U \in \mathscr{U}\left(X^{\wedge}\right) \Longleftrightarrow \exists D \in \mathscr{B} \mathscr{D}(X): \quad D \subseteq U .
$$


В силу предложения 0.8 и леммы 0.2 , система $\mathscr{B} \mathscr{D}(X)$ действительно является локальной базой некоторой локально выпуклой топологии на $X$, поэтому наше определение корректно. Из леммы 0.2, (v) следует, что

$$
\mathscr{B} \mathscr{U}(X) \subseteq \mathscr{B} \mathscr{D}(X) \subseteq \mathscr{B} \mathscr{U}\left(X^{\wedge}\right)
$$

поэтому топология $X^{\wedge}$ мажорирует топологию $X$. Получаюшееся таким образом отображение $X^{\wedge}$ в $X$ обозначается $\wedge_{X}: X^{\wedge} \rightarrow X$.

Лемма 1.18. Отображение $\wedge_{X}: X^{\wedge} \rightarrow X$ является точным наложением, причем:

(i) $X$ псевдонасыщено тогда и только тогда, когда $\wedge_{X}: X^{\wedge} \rightarrow X-$ изоморфизм;

(ii) для любого морфизма ЛВП $\varphi: Y \rightarrow X$ найдется единственный морфизм ЛВП $\varphi^{\wedge}: Y^{\wedge} \rightarrow X^{\wedge}$, замыкающий диаграмму

Здесь важно проверить, что топология действительно не меняется на вполне ограниченных множествах - это вытекает из теоремы 0.7 .

Отсюда следует, что если $\varphi: Y \rightarrow X-$ морфизм из псевдонасышенного ЛВП $Y$, то сушествует единственный морфизм $Y \rightarrow X^{\wedge}$, замыкаюший диаграмму

(b) Проективный ряд. Проективным рядом локально выпуклого пространства $X$ мы называем трансфинитную последовательность локально выпуклых пространств $\left\{X_{\omega} ; \omega \in\right.$ ORD $\}$ (занумерованных ординальными числами - см. [13]) с системой морфизмов ЛВП $\left\{\pi_{\varkappa}^{\iota}: X^{\varkappa} \rightarrow X^{\iota}, \iota<\varkappa \in \mathrm{ORD}\right\}$, удовлетворяющей следующим условиям:

(i) $X_{0}=X$;

(ii) для любых трех ординалов $\iota<\varkappa<\lambda$ коммутативна диаграмма 
(iii) если $\lambda$ - изолированный ординал, т.е. существует ординал $\varkappa$ со свойством (1.12), то сушествует изоморфизм $X_{\lambda}=X_{\varkappa+1} \cong\left(X_{\varkappa}\right)^{\wedge}$ такой, что

(iv) если $\lambda$ - предельный ординал, т.е. (1.12) не выполняется ни для какого $\varkappa$, то существует изоморфизм меж ду $X_{\lambda}$ и локально выпуклым проективным пределом системы $\left\{X_{\iota} ; \iota<\lambda, \iota \rightarrow \lambda\right\}$ такой, что для всякого $\varkappa<\lambda$ коммутативна диаграмма

в которой $\rho_{\varkappa}-$ естественная проекция проективного предела в элемент $X^{\varkappa}$ проективной системы $\left\{X^{\iota} ; \iota<\lambda\right\}$.

Как и в п. 1.3, последовательно доказываются пять лемм.

Лемма 1.19. Всякое ЛВП Х обладает проективным рядом.

Лемма 1.20. Все морфизмы $\pi_{\varkappa}^{\iota}: X_{\iota} \rightarrow X_{\varkappa}$ проективного ряда произвольного данного ЛВП $X$ являются точными наложениями.

Лемма 1.21. Проективный ряд данного ЛВП $X$ определяется однозначно $с$ точностью до изоморфизма.

Это означает, что если $\left\{X_{\omega}, \pi_{\varkappa}^{\iota}\right\}$ и $\left\{\tilde{X}_{\omega}, \tilde{\pi}_{\varkappa}^{\iota}\right\}$ - два проективных ряда для $X$, то сушествует система изоморфизмов $X_{\omega}=\widetilde{X}_{\omega}$ такая, что коммутативна диаграмма

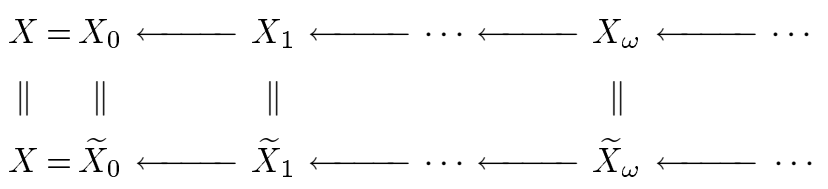

Лемма 1.22. Проективный ряд $\left\{X_{\omega}, \pi_{\varkappa}^{\iota}\right\}$ всякого ЛВП $X$ стабилизируется: существует ординал $\mu$ такой, что

$$
\forall \omega>\mu \quad X_{\omega}=X_{\mu}
$$

(т.е. $\pi_{\omega}^{\mu}: X_{\omega} \rightarrow X_{\mu}$ является изоморфизмом при $\omega>\mu$ ). 
ДоКАЗАТЕЛЬСтво. Из свойств функтора $\wedge$ следует, что проективный ряд можно представлять себе как упорядоченную в сторону усиления систему топологизаций пространства $X$, причем все эти топологии мажорируются топологией насышения:

$$
X=X_{0} \leftarrow X_{1} \leftarrow \cdots \leftarrow X_{\omega} \leftarrow \cdots \leftarrow X^{\mathbf{\Lambda}} .
$$

Поскольку различных топологизаций на $X$ имеется не больше, чем некоторый кардинал $\mathfrak{m}$, который можно оценить, например, неравенством $\mathfrak{m} \leqslant 2^{2^{\operatorname{card}(X)}}$, мы получаем, что по крайней мере начиная с номера $\mu=\mathfrak{m}$ все пространства $X_{\omega}$ совпадают.

Лемма 1.23. Пусть $\left\{X_{\omega} ; \omega \in \mathrm{ORD}\right\}$ - проективньий ряд для ЛВП $X,\left\{Y_{\omega} ; \omega \in\right.$ ORD $\}$ - проективный ряд для ЛВП $Y$. Тогда для любого морфизма $\varphi: Y \rightarrow$ $X$ найдется единственная последовательность морфизмов $\left\{\varphi_{\omega}: Y_{\omega} \rightarrow X_{\omega} ; \omega \in\right.$ ORD\} такая, что коммутативна следующая диаграмма:

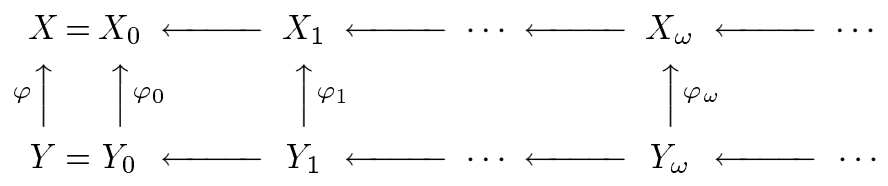

ДокАЗАТЕЛЬСтво. Вначале полагаем $\varphi_{0}=\varphi$. Затем, если для некоторого ординала $\lambda$ все $\left\{\varphi_{\varkappa} ; \varkappa<\lambda\right\}$ уже построены, рассматриваются два случая:

1) если $\lambda$ - изолированный ординал, т.е. выполняется (1.12), то с помошью леммы 1.18 полагаем

$$
\varphi_{\lambda}=\varphi_{\varkappa+1}=\left(\varphi_{\varkappa}\right)^{\wedge}
$$

2 ) если же $\lambda$-предельный ординал, то диаграмма (1.27) будет означать, что для $X_{\omega}$ и $Y_{\omega}$, реализованных как системы топологизаций на $X$ и $Y, X_{\lambda}$ и $Y_{\lambda}$ будут в точности верхними гранями топологий $\left\{X_{\varkappa} ; \varkappa<\lambda\right\}$ и $\left\{Y_{\varkappa} ; \varkappa<\lambda\right\}$, поэтому непрерывность отображений $\left\{\varphi_{\varkappa} ; \varkappa<\lambda\right\}$ влечет за собой непрерывность отображения $\varphi_{\lambda}$.

Лемма доказана.

(c) Конструкция $X^{\Delta}$. Рассмотрим произвольное ЛВП $X$. Его проективный ряд $\left\{X_{\omega} ; \omega \in \mathrm{ORD}\right\}$ по лемме 1.22 стабилизируется. Пусть $\mu$ - ординал, на котором происходит эта стабилизация. Положив

$$
X^{\Delta}=X_{\mu}, \quad \Delta_{X}=\pi_{\mu}^{0},
$$

получим, что, во-первых, пространство $X^{\Delta}$ псевдонасьшено, потому что

$$
\left(X^{\Delta}\right)^{\wedge}=\left(X_{\mu}\right)^{\wedge}=X_{\mu+1}=X_{\mu}=X^{\Delta},
$$

во-вторых, если $X$ псевдонасышено, то по лемме $1.18\left(\pi_{1}^{0}: X_{1} \rightarrow X_{0}\right)=\left(\wedge_{X}: X^{\wedge} \rightarrow\right.$ $X)$ - изоморфизм, откуда следует, что в проективном ряде $\left\{X^{\omega}, \pi_{\varkappa}^{\iota}\right\}$ все морфизмы $\pi_{\varkappa}^{\iota}$ являются изоморфизмами. В частности, $\Delta_{X}=\pi_{\mu}^{0}$ тоже будет изоморфизмом. Наконец, в-третьих, сушествование и единственность морфизма $\varphi^{\Delta}$ в диаграмме (1.20) следует из леммы 1.23 .

Таким образом доказана теорема 1.16. Теорема 1.17 следует из леммы 1.23. 
1.5. Таблица инвариантов. Как известно, полнота сохраняется при переходе к замкнутому подпространству, прямой сумме, прямому произведению и проективному пределу в категории локально выпуклых пространств. Теми же свойствами, как легко заметить, обладает псевдополнота. Двойственным образом оказывается, что псевдонасышенность сохраняется при переходе к (отделимому) факторпространству, прямому произведению, прямой сумме и индуктивному пределу в категории ЛВП.

Напротив, псевдополнота не сохраняется при переходе к факторпространству и локально выпуклому индуктивному пределу, а псевдонасьщенность не сохраняется при переходе к подпространству и проективному пределу.

Наконец, оба свойства - псевдополнота и псевдонасьшенность - сохраняются под действием шести описанных в этом параграфе операций: $\vee, \wedge, \nabla, \Delta, \boldsymbol{\nabla}, \boldsymbol{\Delta}$.

Наглядно все эти закономерности удобно отразить в виде следуюшей таблицы инвариантов.

ТАБЛИЦА ИНВАРИАНТОВ

\begin{tabular}{|c|c|c|}
\hline Операция & Псевдополнота & Псевдонасьшенность \\
\hline$X^{\vee}$ (заготовка к псевдопополнению) & + & $\oplus$ \\
\hline$X^{\wedge}$ (заготовка к псевдонасьшению) & $\oplus$ & + \\
\hline$X^{\nabla}$ (псевдопополнение) & + & $\oplus$ \\
\hline$X^{\triangle}$ (псевдонасышение) & $\oplus$ & + \\
\hline$X^{\mathbf{v}}$ (пополнение) & + & $\oplus$ \\
\hline$X^{\mathbf{\Delta}}$ (насышение) & $\oplus$ & + \\
\hline$\prod_{i \in I} X_{i}$ (прямое произведение) & + & + \\
\hline$\sum_{i \in I} X_{i}$ (прямая сумма) & + & + \\
\hline $\lim _{\longleftarrow} X_{i}$ (проективный предел) & + & $\ominus$ \\
\hline$\stackrel{\lim }{\longrightarrow} X_{i}$ (индуктивный предел) & $\ominus$ & + \\
\hline$Y \subseteq X$ (замкнутое подпространство) & + & $\ominus$ \\
\hline$X / Y$ (отделимое факторпространство) & $\ominus$ & + \\
\hline
\end{tabular}

Кружками здесь обведены минусы и плюсы, требующие проверки (остальные ячейки таблицы мы считаем очевидными). Поскольку минусы в дальнейшем нам не понадобятся, мы не будем их доказывать, отметив только, что все они следуют из примера квазиполного бочечного пространства, обладающего не секвенциально полным отделимым факторпространством [14]. Доказательство плюсов содержится в следующих двух предложениях.

Предложение 1.24. Если $X$ псевдополно, то $X^{\wedge}, X^{\Delta}$ и $X^{\Delta}$ также псевдополнъь. 
(По лемме 1.18 , теореме 1.17 и теореме $1.4 \wedge, \triangle$ и $\mathbf{\Delta}$ сохраняют топологию на вполне ограниченных множествах.)

Предложение 1.25. Если $X$ псевдонасыщено, то $X^{\vee}, X^{\nabla}$ и $X^{\nabla}$ также псевдонасыщены.

Это следует из того, что $\vee_{X}: X \rightarrow X^{\vee}, \nabla_{X}: X \rightarrow X^{\nabla}$ и $\mathbf{\nabla}_{X}: X \rightarrow X^{\mathbf{v}}$ - плотные вложения. Действительно, если $D \in \mathscr{B} \mathscr{D}\left(X^{\vee}\right)$, то $D \cap X \in \mathscr{B} \mathscr{D}(X)$, откуда в силу псевдонасышенности $X$ имеем $D \cap X$ - окрестность нуля в $X$. Значит, замыкание $\overline{D \cap X}$ в пространстве $X^{\vee}$ является окрестностью нуля в $X^{\vee}$. Но $\overline{D \cap X} \subseteq \bar{D}=D$ и, значит, $D$ - тоже окрестность нуля в $X^{\vee}$. Аналогично рассматриваются случаи $X^{\nabla}$ и $X^{\nabla}$.

\section{§ 2. Функтор сопряжения $\star$}

Сопря⿻сенным пространством $X^{\star}$ к ЛВП $X$ над $\mathbb{C}$ мы называем пространство линейных непрерывных функционалов $f: X \rightarrow \mathbb{C}$, наделенное топологией равномерной сходимости на вполне ограниченных множествах в $X$. Если $\varphi: X \rightarrow Y-$ морфизм ЛВП, то формула

$$
\varphi^{\star}(g)=g \circ \varphi
$$

определяет, как легко заметить, морфизм сопряженных пространств $\varphi^{\star}: Y^{\star} \rightarrow X^{\star}$, который мы называем сопряжсенным морфизмом. Отображение $\varphi \mapsto \varphi^{\star}$ является контравариантным функтором в категории ЛВП, и мы называем его функтором сопряжения.

2.1. Двойственность между вполне ограниченными и емкими множествами. Если $B \subseteq X$ и $F \subseteq X^{\star}$ - произвольные множества, то символами $B^{\circ}$ и ${ }^{\circ} F$ обозначаются их (прямая и обратная) поляры (в $X^{\star}$ и $\left.X\right)$ :

$$
B^{\circ}=\left\{f \in X^{\star}:|f|_{B}:=\sup _{x \in B}|f(x)| \leqslant 1\right\}, \quad{ }^{\circ} F=\left\{x \in X:|x|_{F}:=\sup _{f \in F}|f(x)| \leqslant 1\right\} .
$$

Теорема 2.1. Пусть $X-$ произвольное ЛВП. Тогда:

(a) если $B \subseteq X$ вполне ограничено, то $B^{\circ} \subseteq X^{\star}$ емкое;

(b) если $B \subseteq X$ емкое, то $B^{\circ} \subseteq X^{\star}$ вполне ограничено;

(c) если $F \subseteq X^{\star}$ вполне ограничено, то ${ }^{\circ} F \subseteq X$ емкое;

(d) если $F \subseteq X^{\star}$ емкое, то ${ }^{\circ} F \subseteq X$ вполне ограничено.

Для доказательства нам понадобятся несколько вспомогательных утверждений. Условимся символом $X^{\prime}$ обозначать векторное пространство линейных непрерывных функционалов на $X$ (без топологии).

Лемма 2.2. На всяком вполне ограниченном множестве $S \subseteq X \quad X^{\prime}$-слабая топология совпадает с индуцированной из $X$. Поэтому, в частности, $S \in \mathscr{S}(X)$ тогда и только тогда компактно в $X$, когда оно $X^{\prime}$-слабо компактно.

ДокАЗАтЕльство. Рассмотрим пополнение $X^{\boldsymbol{\nabla}}$ пространства $X$. Системы линейных непрерывных функционалов для $X$ и $X^{\mathbf{\nabla}}$ одинаковы. Замыкание $S^{\mathbf{\nabla}}$ множества $S \in \mathscr{S}(X)$ в $X^{\boldsymbol{\nabla}}$ будет компактом в $X^{\boldsymbol{\nabla}}$, на котором функционалы $X^{\prime}=\left(X^{\mathbf{\nabla}}\right)^{\prime}$ индуцируют хаусдорфову топологию, мажорируемую исходной. В силу известного свойства компактов [12] эта топология совпадает с исходной на $S^{\mathbf{v}}$, а значит, и на $S \subseteq S^{\mathbf{v}}$. 
Лемма 2.3. Пусть топология ЛВП $У$ является проективной относительно семейства линейных отображений $\pi_{i}: Y \rightarrow Y_{i}(i \in I)$ в некоторую систему локально выпуклых пространств $\left\{Y_{i} ; i \in I\right\}$. Тогда множество $T \subseteq Y$ будет вполне ограничено в $Y$ в том и только том случае, если для всякого $i \in I$ образ $\pi_{i}(T)$ вполне ограничен в $Y_{i}$.

Необходимость этого условия очевидна, а достаточность доказывается с помощью леммы 0.5: если $\pi_{i}(T)$ вполне ограничено в $Y_{i}$ для любого $i \in I$, то для всякой окрестности нуля $V_{i} \subseteq Y_{i}$ прообраз $\pi_{i}^{-1}\left(V_{i}\right)$ будет емким по отношению к $T$ множеством. Отсюда следует, что если зафиксировать окрестности нуля $V_{i_{1}} \subseteq Y_{i_{1}}, \ldots, V_{i_{n}} \subseteq Y_{i_{n}}$, то их общий прообраз $\bigcap_{k=1}^{n} \pi_{i_{k}}^{-1}\left(V_{i_{k}}\right)$ будет емким по отношению к $T$ множеством, и поскольку все такие множества образуют локальную базу в $Y$, то $T$ должно быть вполне ограничено.

Лемма 2.4 (критерий полной ограниченности в сопряженном пространстве). Множество функционалов $F \subseteq X^{\star}$ вполне ограничено в $X^{\star}$ тогда и только тогда, когда F равностепенно непрерывно на каждом вполне ограниченном множестве $S \subseteq X$

ДокАзАТЕЛьство. Каждому $S \in \mathscr{B} \mathscr{S}(X)$ поставим в соответствие компакт $S^{\mathbf{v}}$, являющийся пополнением вполне ограниченного равномерного пространства $S$ (см. [12, п. 8.3.16]). Пусть $\boldsymbol{\nabla}_{S}: S \rightarrow S^{\mathbf{\nabla}}$ - соответствующее вложение. Всякий функционал $f \in X^{\star}$ равномерно непрерьвен на $S$ и, значит, на $S^{\mathbf{\nabla}}$, поэтому ограничение $\left.f\right|_{S}$ можно (однозначно) продолжить до непрерьвной функции $f_{S}^{\mathbf{\nabla}}: S^{\mathbf{v}} \rightarrow \mathbb{C}$ :

Таким образом, возникает линейное отображение $\pi_{S}: f \mapsto f_{S}^{\boldsymbol{\nabla}}$ пространства $X^{\star}$ в банахово пространство $\mathscr{C}\left(S^{\mathbf{v}}\right)$ непрерывных функций на компакте $S^{\boldsymbol{\nabla}}$ (с топологией равномерной сходимости на $S^{\mathbf{v}}$ ).

Если заставить $S$ меняться, пробегая $\mathscr{B} \mathscr{S}(X)$, то топология $X^{\star}$ окажется проективной относительно системы линейных отображений $\pi_{S}: X^{\star} \rightarrow \mathscr{C}\left(S^{\mathbf{v}}\right)$. Значит, в силу леммы 2.3 множество $F \subseteq X^{\star}$ вполне ограничено в $X^{\star}$ тогда и только тогда, когда для всякого $S \in \mathscr{B} \mathscr{S}(X)$ его образ $F_{S}^{\mathbf{\nabla}}=\pi_{S}(F)$ вполне ограничен в $\mathscr{C}\left(S^{\mathbf{v}}\right)$. По теореме Асколи $[12$, п. 8.2.10] это эквивалентно условиям:

$-F_{S}^{\mathbf{\nabla}}$ равностепенно непрерывно на $S^{\mathbf{v}}$;

$-\forall x \in S^{\boldsymbol{\nabla}} F_{S}^{\mathbf{\nabla}}(x)$ ограничено в $\mathbb{C}$.

Их можно сначала ослабить, заменив $S^{\mathbf{v}}$ на $S$ :

- $F$ равностепенно непрерывно на $S$;

- $\forall x \in S F(x)$ ограничено в $\mathbb{C}$.

А затем второе условие вовсе можно отбросить, потому что если $F$ равностепенно непрерывно на $S$, то $\forall x \in S \quad F$ равностепенно непрерывно на отрезке [0, $x]$ (именно для этого $S$ выбиралось выпуклым и уравновешенным), а отсюда в силу линейности 
функционалов $f \in F$ следует, что $F(x)$ ограничено в $\mathbb{C}$. Мы доказали лемму 2.4, считая, что $S \in \mathscr{B} \mathscr{S}(X)$. Отсюда уже следует, что $S$ может быть любым: $S \in \mathscr{S}(X)$.

Лемма 2.5. Поляра $U^{\circ}$ всякой окрестности нуля $U$ в $X$ является компактом в сопряжсенном пространстве $X^{\star}$.

ДокАЗАТЕЛЬСтво. Из леммы 2.4 следует, что $U^{\circ}$ вполне ограничено в $X^{\star}$. С другой стороны, очевидно, что $U^{\circ}$ полно как равномерное пространство. Значит [12, п. 8.3.16], $U^{\circ}$ является компактом.

Теперь мы можем доказать теорему 2.1 .

1. Если $B \in \mathscr{S}(X)$, то по определению топологии $X^{\star}$ имеем $B^{\circ} \in \mathscr{U}\left(X^{\star}\right) \subseteq \mathscr{D}\left(X^{\star}\right)$.

2. Пусть $B \in \mathscr{D}(X)$. Поскольку поляры $B$ и его замкнутой выпуклой уравновешенной оболочки совпадают, можем считать, что $B \in \mathscr{B} \mathscr{D}(X)$. Тогда по теореме $0.7 B$ будет массивно в нуле:

$$
\forall S \in \mathscr{B} \mathscr{S}(X) \quad \exists U \in \mathscr{B} \mathscr{U}(X): \quad U \cap S \subseteq B .
$$

Переходя к поляре и используя леммы 2.5 и $0.9\left(G=S \subseteq X=P, H=U^{\circ} \subseteq X^{\prime}=Q\right)$, получаем

$$
\forall S \in \mathscr{B} \mathscr{S}(X) \quad \exists U \in \mathscr{B} \mathscr{U}(X): \quad B^{\circ} \subseteq(U \cap S)^{\circ} \subseteq U^{\circ}+S^{\circ} .
$$

Здесь $S^{\circ}$ - базисная окрестность нуля в $X^{\star}$, а $U^{\circ}$-компакт в $X^{\star}$ (по лемме 2.5). Значит,

$$
\forall V \in \mathscr{B} \mathscr{U}\left(X^{\star}\right) \quad \exists K \in \mathscr{B} \mathscr{K}\left(X^{\star}\right): \quad B^{\circ} \subseteq K+V .
$$

Это равносильно условию $B^{\circ} \in \mathscr{S}\left(X^{\star}\right)$ в силу предложения 0.4 .

3. Пусть $F \in \mathscr{S}\left(X^{\star}\right)$. Тогда по лемме $2.4 F$ равностепенно непрерьвно на каждом $S \in \mathscr{S}(X)$. Отсюда

$$
\forall S \in \mathscr{B} \mathscr{S}(X) \quad \exists U \in \mathscr{B} \mathscr{U}(X): \quad U \cap S \subseteq{ }^{\circ} F,
$$

т.е. ${ }^{\circ} F$ массивно в нуле и, значит, является емким в $X$.

4. Пусть $F \in \mathscr{D}\left(X^{\star}\right)$. Поскольку обратные поляры $F$ и его замкнутой выпуклой уравновешенной оболочки совпадают, можно считать, что $F \in \mathscr{B} \mathscr{D}\left(X^{\star}\right)$. Зафиксируем окрестность нуля $U \in \mathscr{B} \mathscr{U}(X)$ и рассмотрим поляру $T=U^{\circ} \in \mathscr{B}\left(X^{\star}\right)$. По лемме 2.5 $T \in \mathscr{B} \mathscr{K}\left(X^{\star}\right)$. Значит, по теореме 0.7

$$
\exists V \in \mathscr{B} \mathscr{U}\left(X^{\star}\right): \quad V \cap T \subseteq F .
$$

Заметим теперь, что на компакте $T=U^{\circ} \subseteq X^{\star} X$-слабая топология хаусдорфова и мажорируется $X^{\star}$-топологией. Значит [12, п. 3.1.14], эти топологии на $T$ совпадают. Поэтому должен существовать конечномерный компакт $K \in \mathscr{B} \mathscr{K}(X)$ такой, что

$$
K^{\circ} \cap T \subseteq V \cap T \subseteq F .
$$

Воспользуемся теперь леммой $0.9\left(G=T \subseteq X^{\star}=P, H=K \subseteq X=Q\right)$ :

$$
{ }^{\circ} F \subseteq{ }^{\circ}\left(K^{\circ} \cap T\right) \subseteq{ }^{\circ}\left(K^{\circ}\right)+{ }^{\circ} T \subseteq K+{ }^{\circ} T \subseteq K+U .
$$

Мы получили, что

$$
\forall U \in \mathscr{B} \mathscr{U}(X) \quad \exists K \in \mathscr{B} \mathscr{K}(X): \quad{ }^{\circ} F \subseteq K+U .
$$

По предложению 0.4 это означает, что ${ }^{\circ} F \in \mathscr{B} \mathscr{S}(X)$. Теорема 2.1 доказана. 
2.2. Кополнота и связь между насышенностью и псевдонасышенностью. Назовем ЛВП $X$ кополныцм, если его сопряженное пространство $X^{\star}$ полно.

Предложение 2.6. Для ЛВП Х следующие условия әквивалентны:

(i) пространство $X$ кополно:

(ii) всякий линейный функционал $f: X \rightarrow \mathbb{C}$, непрерывный на каждом вполне ограниченном множестве $S \subseteq X$, непрерывен на всем $X$.

ДокАЗАтЕЛьСтво. Импликация (ii) $\Rightarrow$ (i) очевидна. Докажем обратную(i) $\Rightarrow$ (ii). Пусть $X$ кополно, т.е. $X^{\star}$ полно. Обозначим через $X^{\natural}$ пространство всех линейных функционалов $f: X \rightarrow \mathbb{C}$, непрерывных на каждом $S \in \mathscr{B} \mathscr{S}(X)$. Если наделить $X^{\natural}$ топологией равномерной сходимости на вполне ограниченных множествах $S \in X$, мы получим вложение локально выпуклых пространств $X^{\star} \subseteq X^{\natural}$. При этом $X^{\star}$ полно и, значит, замкнуто в $X^{\natural}$. Поэтому для доказательства равенства $X^{\star}=X^{\natural}$ нам нужно только проверить, что $X^{\star}$ плотно в $X^{\natural}$.

Возьмем для этого функционал $f \in X^{\natural}$ и зафиксируем $S \in \mathscr{B} \mathscr{S}(X)$ и $\varepsilon>0$. Покажем, что сушествует $g \in X^{\star}$ такой, что

$$
|f-g|_{S} \leqslant \varepsilon .
$$

Функционал $f$ непрерывен на $S$, поэтому сушествует окрестность нуля $V \in \mathscr{B} \mathscr{U}(X)$ такая, что $|f|_{S \cap V} \leqslant \varepsilon$, т.е. $f \in \varepsilon(S \cap V)_{X}^{\circ}$. В силу леммы 2.2 найдется $X^{\star}$-слабая окрестность нуля $W$ в $X$ такая, что $S \cap W \subseteq S \cap V$. При этом можно считать, что $W$ выпукла, уравновешена и $X^{\star}$-слабо замкнута, т.е. $W={ }^{\circ} K$ для некоторого конечномерного компакта $K \in \mathscr{B} \mathscr{K}\left(X^{\star}\right)\left(K \subseteq X^{\star} \subseteq X^{\natural}\right)$ :

$$
f \in \varepsilon(S \cap V)_{X^{\natural}}^{\circ} \subseteq \varepsilon(S \cap W)_{X^{\natural}}^{\circ} \subseteq \varepsilon\left(S \cap{ }^{\circ} K\right)_{X^{\natural}}^{\circ} .
$$

Воспользуемся теперь леммой $0.9\left(G=S \subseteq X=P, H=K \subseteq X^{\natural}=Q\right)$ :

$$
\left(S \cap{ }^{\circ} K\right)_{X^{\natural}}^{\circ} \subseteq S_{X^{\natural}}^{\circ}+K \text {. }
$$

Значит, $f \in \varepsilon S_{X^{\natural}}^{\circ}+\varepsilon K$. Таким образом, существует $g \in \varepsilon K \subseteq X^{\star}$ такой, что $f-$ $g \in \varepsilon S_{X}^{\circ}$, т.е. выполняется $(2.1)$.

Теорема 2.7 (о связи между насышенностью и псевдонасышенностью). ЛВП $X$ насыщено тогда и только тогда, когда оно псевдонасыщено и кополно.

ДокАЗАТЕльство. Необходимость очевидна, докажем достаточность. Пусть $X$ псевдонасышено и кополно. Рассмотрим насышение $X^{\boldsymbol{\Delta}}$ пространства $X$. Из кополноты $X$ следует, что сопряженные пространства у $X$ и $X^{\mathbf{\Delta}}$ совпадают:

$$
X^{\star}=\left(X^{\mathbf{\Delta}}\right)^{\star} .
$$

Пусть $M$ - предкомпактно-замкнутое выпуклое уравновешенное массивное в нуле множество в $X$, т.е. $M \in \mathscr{P} \mathscr{M}(X)$. Тогда в силу (1.4) $M \in \mathscr{B} \mathscr{U}\left(X^{\mathbf{\Delta}}\right)$, а отсюда в силу (2.2) $M \in \mathscr{B}(X)$. Значит, $M \in \mathscr{B} \mathscr{M}(X)=\mathscr{B} \mathscr{U}(X)$. Таким образом, $\mathscr{P} \mathscr{M}(X) \subseteq \mathscr{B} \mathscr{U}(X)$, т.е. $X$ насыщено.

2.3. Критерии насышенности и псевдонасышенности. Из теоремы 2.1 следует

Теорема 2.8 (критерий псевдонасьщенности). ЛВП $X$ тогда и только тогда псевдонасыщено, когда всякое множсество линейных непрерывных функчионалов 
$F \subseteq X^{\prime}$, равностепенно непрерывное на каждом вполне ограниченном множестве $S \subseteq X$, равностепенно непрерывно на всем $X$.

ДоказАтельство. Необходимость. Рассмотрим обратную поляру ${ }_{\circ} F \subseteq X$. В силу леммы 2.4 и теоремы 2.1 , (с) она будет замкнутым выпуклым уравновешенным емким множеством в $X$. Поэтому если $X$ псевдонасьшено, то $॰ F \subseteq X$ есть окрестность нуля, т.е. $F$ равностепенно непрерьвно на $X$.

Достаточность. Пусть $D \in \mathscr{B} \mathscr{D}(X)$. Поляра $F=D^{\circ}$ будет по теореме 2.1 , (b) и лемме 2.4 множеством функционалов из $X^{\prime}$, равностепенно непрерывным на каждом $S \in \mathscr{S}(X)$. Если это равносильно равностепенной непрерывности $F$ на всем $X$, то обратная поляра $D={ }_{\circ} F$ будет окрестностью нуля в $X$.

Теоремы 2.8 и 2.7 вместе дают следуюшую теорему.

Теорема 2.9 (критерий насьпенности). ЛВП Х тогда и только тогда насыщено, когда всякое множество линейных функционалов $F \subseteq X^{\prime}$ (не предполагаемых заранее непрерывными), равностепенно непрерывное на каждом вполне ограниченном множестве $S \subseteq X$, равностепенно непрерывно на всем $X$.

2.4. Отображение $i_{X}: X \rightarrow X^{\star \star}$. Вторым сопряженным пространством $X^{\star \star}$ к ЛВП $X$ мы называем сопряженное к первому сопряженному:

$$
X^{\star \star}=\left(X^{\star}\right)^{\star}
$$

(каждая звездочка 夫 означает, что берется топология равномерной сходимости на вполне ограниченных множествах). Формула

$$
i_{X}(x)(f)=f(x)
$$

определяет естественное отображение $i_{X}: X \rightarrow X^{\star \star}$.

Теорема 2.10. Для всякого ЛВП $Х$ отображсение $i_{X}: X \rightarrow X^{\star \star}$ инвективно, открыто и имеет плотный в $X^{\star \star}$ образ.

ЗАмЕчАниЕ. Это отображение не обязано быть непрерывным. Его открытость означает, что

$$
\forall U \in \mathscr{U}(X) \quad \exists V \in \mathscr{U}\left(X^{\star \star}\right): \quad i_{X}(U) \supseteq V \cap i_{X}(X) .
$$

Для доказательства заметим, что отображение $i_{X}: X \rightarrow X^{\star \star}$ переводит всякое множество $B \subseteq X$ в его вторую поляру $B^{\circ \circ}=\left\{y \in X^{\star \star}: \forall f \in B^{\circ}|y(f)| \leqslant 1\right\}$. Поэтому определено отображение $i_{B}: B \rightarrow B^{\circ \circ}$.

Лемма 2.11. Пусть $S \in \mathscr{B} \mathscr{S}(X) ;$ тогда вторая поляра $S^{\circ \circ}$ является компактом в $X^{\star \star}$, а отображсение $i_{S}: S \rightarrow S^{\circ \circ}$ является вложением топологических пространств с плотным образом.

Действительно, первая поляра $S^{\circ}$ будет окрестностью нуля в $X^{\star}$ в силу выбора топологии $X^{\star}$, а вторая поляра $S^{\circ \circ}$ поэтому будет компактом в $X^{\star \star}$ по лемме 2.5 . По лемме 2.2 топология $S$ совпадает с $X^{\prime}$-слабой. С другой стороны, топология $S^{\circ \circ}$ также совпадает с $X^{\prime}$-слабой, потому что $X^{\prime}$-слабая топология является хаусдорфовой топологией на компакте $S^{\circ}$, мажорируемой исходной топологией этого компакта (см. [12]). Значит, $i_{S}: S \rightarrow S^{\circ \circ}$ является вложением. С другой стороны, по теореме о биполяpe [11] $i(S)$ плотно в $S^{\circ \circ}$ в смысле $X^{\prime}$-слабой топологии, поэтому $i(S)$ плотно в $S^{\circ \circ}$ в смысле топологии пространства $X^{\star \star}$. 
Лемма 2.12. Для всякого ЛВП Х справедливо равенство (множеств)

$$
X^{\star \star}=\bigcup_{S \in \mathscr{B} \mathscr{S}(X)} S^{\circ \circ} .
$$

ДоказАТЕльство. Возьмем $z \in X^{\star \star}$. Поскольку функционал $z: X^{\star} \rightarrow \mathbb{C}$ непрерывен, он должен быть ограничен на некоторой базисной окрестности нуля $V=S^{\circ}$, $S \in \mathscr{S}(X)$. Пошевелив в случае необходимости $S \in \mathscr{S}(X)$, можно в качестве ограничивающей константы взять единищу. Таким образом, $|z|_{V} \leqslant 1$, т.е. $z \in V^{\circ}=S^{\circ \circ}$.

Из лемм 2.11 и 2.12 следует

Лемма 2.13. Для всякого ЛВП $Х$ любой вектор $z \in X^{\star \star}$ является пределом в $X^{\star \star}$ некоторой направленности из $i_{X}(X)$ :

$$
z=\lim _{\nu \rightarrow \infty} i_{X}\left(x_{\nu}\right)
$$

такой, что $\left\{x_{\nu}\right\}$ вполне ограничено в $X, a\left\{i_{X}\left(x_{\nu}\right)\right\}$ вполне ограничено в пространстве $X^{\star \star}$.

ДОКАЗАТЕЛЬСТВО ТЕОРЕмЫ 2.10. Инъективность $i_{X}$ очевидна. Плотность $i_{X}(X)$ в $X^{\star \star}$ следует из леммы 2.13. Проверим открытость. Пусть $U \in \mathscr{B} \mathscr{U}(X)$. Тогда по лемме $2.5 U^{\circ} \in \mathscr{B} \mathscr{K}\left(X^{\star}\right) \subseteq \mathscr{B} \mathscr{S}\left(X^{\star}\right)$ и, значит, $U^{\circ \circ} \in \mathscr{B} \mathscr{U}\left(X^{\star \star}\right)$. Таким образом, $i_{X}(U)=U^{\circ \circ} \cap i_{X}(X)$ будет окрестностью нуля в пространстве $i_{X}(X) \subseteq X^{\star \star}$.

2.5. Двойственность между псевдополнотой и псевдонасыщенностью. Итак, отображение $i_{X}: X \rightarrow X^{\star \star}$ всегда инъективно, открыто и имеет всюду плотный образ. Естественный вопрос о сюръективности и непрерьвности этого отображения, как оказывается, напрямую связан с псевдополнотой и псевдонасьшенностью $X$.

Теорема 2.14. Для произвольного ЛВП Х следующие условия әквивалентны:

(i) пространство $X$ псевдополно;

(ii) отображсние $i_{X}: X \rightarrow X^{\star \star}$ сюрвективно ( $и$, значит, биективно).

ДокАЗАтЕльСтво. По теореме Макки [11] $i_{X}: X \rightarrow X^{\star \star}$ биективно тогда и только тогда, когда сушествует локальная база в $X^{\star}$, состоящая из поляр $T^{\circ} X^{\star}$-слабо компактных множеств $T \subseteq X$. Это означает, что для всякого $S \in \mathscr{B} \mathscr{S}(X)$ должно сушествовать $X^{\star}$-слабо компактное $T \in \mathscr{B}(X)$ такое, что $T^{\circ} \supseteq S^{\circ}$ т.е. $S \subseteq T$. Иными словами, всякое $S \in \mathscr{B} \mathscr{S}(X)$ должно быть $X^{\star}$-слабо компактным и, значит (лемма $2.2)$, просто компактным.

Следствие 2.15. Если пространство X псевдополно, то определено (непрерывное) отобрахсение $i_{X}^{-1}: X^{\star \star} \rightarrow X$, являющееся точным налохсением.

Действительно, из открытости (теорема 2.10) и биективности отображения $i_{X}: X \rightarrow$ $X^{\star \star}$ следует существование и непрерывность обратного отображения $i_{X}^{-1}: X^{\star \star} \rightarrow X$. При этом если $S \in \mathscr{B} \mathscr{S}(X)$, то по лемме 2.11

$$
i_{X}(S)=S^{\circ \circ} \cap i_{X}(X)=S^{\circ \circ} \cap X^{\star \star}=S^{\circ \circ} \in \mathscr{B} \mathscr{S}\left(X^{\star \star}\right),
$$

т.е. сушествует $T=i_{X}(S) \in \mathscr{B} \mathscr{S}\left(X^{\star \star}\right)$ такое, что $i_{X}^{-1}(T)=S$. 
Теорема 2.16. Для произвольного ЛВП $Х$ следующие условия әквивалентны:

(i) пространство $X$ псевдонасыщено;

(ii) отображсение $i_{X}: X \rightarrow X^{\star \star}$ непрерывно (

ДоказАТЕльство. (i) $\Rightarrow$ (ii). Пусть $X$ псевдонасьшено и $V$ - базисная окрестность нуля в $X^{\star \star}$, т.е. $V=F^{\circ}$, где $F \in \mathscr{B} \mathscr{S}\left(X^{\star}\right)$. По теореме 2.1 , (d) множество ${ }^{\circ} F=$ $i^{-1}\left(F^{\circ}\right)=i^{-1}(V)$ должно быть емким, т.е. окрестностью нуля в $X$.

(ii) $\Rightarrow(\mathrm{i})$. Возьмем $D \in \mathscr{B} \mathscr{D}(X)$. По теореме 2.1 , (b) поляра $D^{\circ}$ вполне ограничена в $X^{\star}$, поэтому вторая поляра $D^{\circ \circ}$ есть окрестность нуля в $X^{\star \star}$. Если $i_{X}: X \rightarrow X^{\star \star}$ непрерывно, то $D=i^{-1}\left(D^{\circ \circ}\right)$ будет окрестностью нуля в $X$.

Следствие 2.17. Если пространство $X$ псевдонасыщено, то отображение $i_{X}: X \rightarrow X^{\star \star}$ является плотницм вложением.

Следуюший результат устанавливает двойственность между псевдополными и псевдонасышенными пространствами.

Теорема 2.18. Пусть $Х-$ произвольное ЛВП. Тогда:

- если Х псевдополно, то $X^{\star}$ псевдонасыщено;

- если Х псевдонасыщено, то $X^{\star}$ псевдополно.

ДоКАЗАТЕЛЬСТВО использует следуюшую очевидную формулу:

$$
\forall f \in X^{\star} \quad i_{X^{\star}}(f) \circ i_{X}=f .
$$

1. Пусть $X$ псевдополно, т.е. (теорема 2.14) $i_{X}: X \rightarrow X^{\star \star}$ является биекцией. Тогда определено обратное отображение $i_{X}^{-1}: X^{\star \star} \rightarrow X$, непрерывное в силу следствия 2.15 . Поэтому из формулы $i_{X^{\star}}(f)=f \circ i_{X}^{-1}$ следует, что $i_{X^{\star}}$ есть непрерывное отображение, т.е. (теорема 2.16) $X^{\star}$ псевдонасышено.

2. Пусть $X$ псевдонасышено, т.е. $i_{X}: X \rightarrow X^{\star \star}$ непрерывно (теорема 2.16). Тогда, выбрав $g \in X^{\star \star \star}$ и положив $f=g \circ i_{X}$, мы получим, что $f \in X^{\star}$, причем

$$
i_{X^{\star}}(f) \circ i_{X}=f=g \circ i_{X},
$$

т.е. функционалы $i_{X^{\star}}(f)$ и $g$ совпадают на подпространстве $i_{X}(X)$, плотном в $X^{\star \star}$, в силу теоремы 2.10. Следовательно, они совпадают на всем $X^{\star \star}$. Мы получили, что отображение $i_{X^{\star}}: X^{\star} \rightarrow X^{\star \star \star}$ сюръективно, т.е. (теорема 2.14) $X^{\star \star}$ псевдополно.

\section{§ 3. Двойственность между функторами $\nabla$ и $\triangle$}

3.1. Двойственность между плотными вложениями и точными наложениями. В этом пункте мы доказываем следуюшие две теоремы.

Теорема 3.1. Если $X$ псевдополно и $\varphi: X \rightarrow Y$ - точное наложение, то $\varphi^{\star}: Y^{\star} \rightarrow X^{\star}-$ плотное вложение.

Теорема 3.2. Если $X$ псевдонасыщено и $\varphi: X \rightarrow Y$ - плотное вложсение, то $\varphi^{\star}: Y^{\star} \rightarrow X^{\star}-$ точное наложение.

Их доказательству мы предпошлем несколько лемм. 


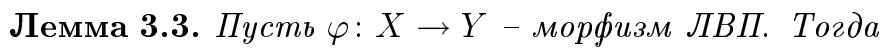

$$
\forall A \subseteq X \quad\{\varphi(A)\}_{Y^{\star}}^{\circ}=\left(\varphi^{\star}\right)^{-1}\left(A_{X^{\star}}^{\circ}\right),
$$

а если $X$ псевдополно, то

$$
\forall B \subseteq Y \quad\left\{\varphi^{-1}(B)\right\}_{X^{\star}}^{\circ}=\overline{\varphi^{\star}\left(B_{Y^{\star}}^{\circ}\right)} .
$$

ДоКАЗАТЕЛЬСтво. Если $A \subseteq X$, то $[\varphi(A)]_{Y^{\star}}^{\circ}=\left\{g \in Y^{\star}:|g|_{\varphi(A)} \leqslant 1\right\}=\left\{g \in Y^{\star}\right.$ : $\left.\left|\varphi^{\star}(g)\right|_{A}=|g \circ \varphi|_{A} \leqslant 1\right\}=\left\{g \in Y^{\star}: \varphi^{\star}(g) \in A_{X^{\star}}^{\circ}\right\}=\left(\varphi^{\star}\right)^{-1}\left(A_{X^{\star}}^{\circ}\right)$. Пусть теперь $X$ псевдополно и $B \subseteq Y$. Тогда по теореме 2.14

$$
\begin{aligned}
{\left[\varphi^{\star}\left(B_{Y^{\star}}^{\circ}\right)\right]_{X^{\star \star}}^{\circ} } & =\left[\varphi^{\star}\left(B_{Y^{\star}}^{\circ}\right)\right]_{X}^{\circ}=\left\{x \in X: \forall f \in \varphi^{\star}\left(B_{Y^{\star}}^{\circ}\right)|f(x)| \leqslant 1\right\} \\
& =\left\{x \in X: \forall g \in B_{Y^{\star}}^{\circ}\left|\varphi^{\star}(g)(x)\right|=|g(\varphi(x))| \leqslant 1\right\} \\
& =\left\{x \in X: \varphi(x) \in\left(B_{Y^{\star}}^{\circ}\right)_{Y}^{\circ}\right\}=\varphi^{-1}\left[\left(B_{Y^{\star}}^{\circ}\right)_{Y}^{\circ}\right] \\
& =\varphi^{-1}\left[\overline{\operatorname{absconv}}_{Y} B\right]=\overline{\operatorname{absconv}}_{X}\left[\varphi^{-1}(B)\right],
\end{aligned}
$$

где $\overline{\mathrm{absconv}}$ - замкнутая выпуклая уравновешеная оболочка. Поскольку $B_{Y^{\star}}^{\circ}$, а значит, и $\varphi^{\star}\left(B_{Y^{\star}}^{\circ}\right)$ выпуклы и уравновешены, имеем

$$
\begin{aligned}
\overline{\varphi^{\star}\left(B_{Y^{\star}}^{\circ}\right)} & =\overline{\operatorname{absconv}}_{X^{\star}} \varphi^{\star}\left(B_{Y^{\star}}^{\circ}\right)=\left\{\left[\varphi^{\star}\left(B_{Y^{\star}}^{\circ}\right)\right]_{X^{\star \star}}^{\circ}\right\}_{X^{\star}}^{\circ} \\
& =\left\{\overline{\operatorname{absconv}}_{X}\left[\varphi^{-1}(B)\right]\right\}_{X^{\star}}^{\circ}=\left\{\varphi^{-1}(B)\right\}_{X^{\star}}^{\circ}
\end{aligned}
$$

Лемма доказана.

Из нее следует

Лемма 3.4. Если $X$ псевдополно и $\varphi: X \rightarrow Y-$ мономорфизм, то $\varphi^{\star}: Y^{\star} \rightarrow$ $X^{\star}-$ эпиморфизм.

Действительно, положив $B=\{0\} \subseteq Y$, мы получим $X^{\star}=\{0\}_{X^{\star}}^{\circ}=$ $\left\{\varphi^{-1}(B)\right\}_{X^{\star}}^{\circ}=\overline{\varphi^{\star}\left(B_{Y^{\star}}^{\circ}\right)}=\overline{\varphi^{\star}\left(Y^{\star}\right)}$.

Лемма 3.5. Если $X$ псевдонасыщено и $\varphi: X \rightarrow Y-$ вложсение, то $\varphi^{\star}: Y^{\star} \rightarrow$ $X^{\star}-$ наложение.

ДоКАЗАТЕЛЬСтво. Пусть $F \in \mathscr{S}\left(X^{\star}\right)$. В силу леммы 2.4 и теоремы $2.8 F$ равностепенно непрерывно на $X$, т.е. все функционалы $f \in F$ подчинены одной полунорме $p$, непрерывной на $X$ :

$$
|f(x)| \leqslant p(x), \quad x \in X, \quad f \in F .
$$

Поскольку $\varphi: X \rightarrow Y$-вложение, полунорма $p$ продолжается до некоторой полунормы $q$, непрерывной на $Y: q \circ \varphi=p$. С помошью теоремы Хана-Банаха для всякого $f \in F$ выберем продолжение $f_{Y} \in Y^{\star}$, подчиненное $q$ :

$$
f_{Y} \circ \varphi=f \quad \& \quad \forall y \in Y \quad\left|f_{Y}(y)\right| \leqslant q(y) .
$$

Множество функционалов $F_{Y}=\left\{f_{Y} ; f \in F\right\}$ подчинено непрерывной полунорме $q$ на $Y$, т.е. равностепенно непрерьвно на $Y$. Значит (лемма 2.4), $F_{Y} \in \mathscr{S}\left(Y^{\star}\right)$. При этом

$$
\varphi^{\star}\left(F_{Y}\right)=F_{Y} \circ \varphi=F .
$$

Лемма доказана.

Следуюшие леммы очевидны. 
Лемма 3.6. Если $\varphi: X \rightarrow Y-$ наложение, то $\varphi^{\star}: Y^{\star} \rightarrow X^{\star}-$ вложсение.

Лемма 3.7. Если $\varphi: X \rightarrow Y-$ эпиморфизм, то $\varphi^{\star}: Y^{\star} \rightarrow X^{\star}-$ мономорфизм.

Теорема 3.1 теперь следует из лемм 3.4 и 3.6 .

ДОКАЗАТЕЛЬСТВо ТЕОРЕМЫ 3.2. Из лемм 3.5 и 3.7 следует, что если $X$ псевдонасьшено и $\varphi: X \rightarrow Y$ - плотное вложение, то $\varphi^{\star}: Y^{\star} \rightarrow X^{\star}-$ биективное наложение. Для доказательства его точности возьмем вполне ограниченное множество $G \subseteq Y^{\star}$. Его образ $F=\varphi^{\star}(G)=G \circ \varphi$ будет вполне ограничен в $X^{\star}$. По лемме 2.4 и теореме $2.8 F$ равностепенно непрерывно на $X$, значит $F$ содержится в поляре $U^{\circ}$ некоторой окрестности нуля $U \subseteq X$. Поскольку $\varphi: X \rightarrow Y$-вложение, сушествует окрестность нуля $V \subseteq Y$ такая, что $U=\varphi^{-1}(V)$. При этом $G \subseteq Y^{\star}$ будет содержаться в поляре $V^{\circ}$.

Заметим теперь, что (по лемме 2.5$) U^{\circ}$ - компакт в $X^{\star}$, а $X$-слабая топология на нем хаусдорфова и мажорируется исходной (т.е. индуцированной из $X^{\star}$ ). Значит, исходная топология $U^{\circ}$ совпадает с $X$-слабой. С другой стороны, поскольку $X$ плотно в $Y$ (в смысле $\varphi$ ), на компакте $V^{\circ} \subseteq Y^{\star} X$-слабая топология тоже хаусдорфова и тоже мажорируется исходной (т.е. индуцированной из $Y^{\star}$ ). Значит, исходная топология $V^{\circ}$ совпадает с $X$-слабой.

Итак, топологии компактов $U^{\circ} \subseteq X^{\star}$ и $V^{\circ} \subseteq Y^{\star}$ совпадают с $X$-слабой топологией, и это значит, что $V^{\circ}$ и $U^{\circ}$ гомеоморфны в смысле биекции $\varphi^{\star}: Y^{\star} \rightarrow X^{\star}$. Следовательно, их подпространства $G \subseteq V^{\circ}$ и $F \subseteq U^{\circ}$ также гомеоморфны относительно $\varphi^{\star}$. Теорема 3.2 доказана.

\section{2. Двойственность между $\vee$ и $\wedge$.}

Лемма 3.8. Для всякого псевдополного ЛВП Х существует (единственный) изоморфизм $X^{\wedge}=X^{\star \star}$ такой, что коммутативна диаграмма

При этом для всякого морфизма псевдополных ЛВП $\varphi: X \rightarrow Y$ будет коммутативна диаграмма 
ДокАЗАТЕЛьСТво. В силу следствия 2.15 отображение $i_{X}^{-1}: X^{\star \star} \rightarrow X$ является точным наложением, и поэтому его можно рассматривать как более тонкую топологизацию $X$, сохраняюшую вполне ограниченные множества. Локальной базой такой топологии будут множества ${ }^{\circ} F, F \in \mathscr{B} \mathscr{S}\left(X^{\star}\right)$, т.е. в силу теоремы 2.1 , (b), (c) множества $D \in \mathscr{B} \mathscr{D}(X)$. Это совпадает с определением $X^{\wedge}$. Дальнейшее ясно.

Лемма 3.9. Для всякого псевдонасыщенного ЛВП Х существует (единственньй) изоморфизм $X^{\vee}=X^{\star \star}$ такой, что коммутативна диаграмма

При этом для всякого морфизма псевдонасыщенных ЛВП $\varphi: X \rightarrow Y$ будет коммутативна диаграмма

Это следует из определения $X^{\vee}$, леммы 2.13 и следствия 2.17 .

Теорема 3.10. Если $X$ псевдополно, то существует (единственныци) изоморфизм $\left(X^{\wedge}\right)^{\star}=\left(X^{\star}\right)^{\vee}$ такой, что коммутативна диаграмма

При этом для всякого морфизма псевдополных ЛВП $\varphi: X \rightarrow Y$ будет коммутативна диаграмма 
ДоКАЗАТЕЛЬСТво. По теореме $2.18 X^{\star}$ будет псевдонасьшено, поэтому

$$
\begin{aligned}
\left(X^{\wedge}\right)^{\star} & =(\text { лемма } 3.8)=\left(X^{\star \star}\right)^{\star}=\left(X^{\star}\right)^{\star \star}=(\text { лемма } 3.9)=\left(X^{\star}\right)^{\vee}, \\
\left(\varphi^{\wedge}\right)^{\star} & =(\text { лемма 3.8) }
\end{aligned}
$$

Теорема 3.11. Если $X$ псевдонасыщено, то существует (единственный) изоморфизм $\left(X^{\vee}\right)^{\star}=\left(X^{\star}\right)^{\wedge}$ такой, что коммутативна диаграмма

При этом для всякого морфизма псевдонасыщенных ЛВП $\varphi: X \rightarrow Y$ будет коммутативна диаграмма

ДокАЗАТЕЛЬСТвО. По теореме $2.18 X^{\star}$ будет псевдополно, поэтому

$$
\begin{aligned}
\left(X^{\vee}\right)^{\star} & =\left(\text { лемма 3.9) }=\left(X^{\star \star}\right)^{\star}=\left(X^{\star}\right)^{\star \star}=(\text { лемма } 3.8)=\left(X^{\star}\right)^{\wedge},\right. \\
\left(\varphi^{\vee}\right)^{\star} & =\left(\text { лемма 3.9) }=\left(\varphi^{\star \star}\right)^{\star}=\left(\varphi^{\star}\right)^{\star \star}=(\text { лемма } 3.8)=\left(\varphi^{\star}\right)^{\wedge} .\right.
\end{aligned}
$$

\section{3. Двойственность между инъективным и проективным рядами.}

Теорема 3.12. Пусть $X$ - псевдополное ЛВП $и\left\{X_{\omega}, \pi\right\}-$ проективный ряд для X. Тогда:

(a) проективный ряд $\left\{X_{\omega}, \pi\right\}$ состоит из псевдополных пространств;

(b) если $\left\{\left(X^{\star}\right)_{\omega}, \sigma\right\}$ - инбективный ряд для сопряжсенного пространства $X^{\star}$, то существует единственная система изоморфизмов

$$
\left(X_{\omega}\right)^{\star}=\left(X^{\star}\right)^{\omega}
$$

такая, что для всякого $\varkappa<\lambda$ коммутативна диаграмма 
(с) для всякого морфизма псевдополных ЛВП $\varphi: X \rightarrow Y$ и любого ординала $\omega$ коммутативна диаграмма

в которой $\left\{Y_{\omega}\right\}-$ произвольный проективный ряд для $Y,\left\{\left(Y^{\star}\right)^{\omega}\right\}-$ произвольньи й ингективный ряд для $Y$, а $\varphi_{\omega} u\left(\varphi^{\star}\right)^{\omega}$ - системы морфизмов, определенные в леммах 1.23 u 1.12 .

ДокАЗАТЕЛЬСтво. Заметим сразу, что в силу таблицы инвариантов п. 1.5 все пространства $\left\{X_{\omega}\right\}$ псевдополны. Проверим, что система $\left(X_{\omega}\right)^{\star}$ является инъективным рядом для $X$, т.е. удовлетворяет условиям (i)-(iv) п. 1.3. Условия (i) и (ii) выполняются автоматически. Докажем (iii). Возьмем изолированный ординал $\lambda$ и рассмотрим тетраэдр

в котором верхний внутренний треугольник есть диаграмма (3.7), а левый внутренний треугольник - диаграмма, сопряженная к (1.26). Определив пунктирную стрелку как композицию морфизмов $X_{\varkappa}^{\star} \longrightarrow X_{\varkappa}^{\wedge \star} \longrightarrow\left(X_{\lambda}\right)^{\star}$, мы получим коммутативную диаграмму (3.13). Пунктирная стрелка, как и другие стороны большого треугольника, будет изоморфизмом, поэтому примыкающий к пунктирной стрелке правый внутренний треугольник есть попросту диаграмма (1.13).

Проверим теперь условие (iv). Для этого возьмем предельный ординал $\lambda$ и покажем, что имеется естественный изоморфизм

$$
\left.\underset{\iota<\lambda}{\lim } X_{\iota}\right)^{\star}=\left(X_{\lambda}\right)^{\star}=\underset{\iota<\lambda}{\lim }\left(X_{\iota}\right)^{\star}
$$

Действительно, если представлять себе $X_{\omega}$ как цепочку топологизаций $\tau_{\omega}$ векторного пространства $X$ (направленную в сторону усиления), то топология $\tau_{\lambda}$ пространства $X_{\lambda}$ будет в точности верхней гранью (объединением) топологий $\tau_{\iota}, \iota<\lambda$. Поэтому формула (3.6), рассматриваемая как равенство множеств, будет означать просто, что функционал $f$ на $X$ тогда и только тогда непрерывен в топологии $\tau_{\lambda}$, когда он непрерывен 
в какой-нибудь топологии $\tau_{\iota}, \iota<\lambda$. С другой стороны, формула (3.6), рассматриваемая как совпадение топологических пространств, означает, что множество $F$ линейных функционалов на $X$ тогда и только тогда является окрестностью нуля в $X$, когда в каждом пространстве $\left(X_{\iota}\right)^{\star}, \iota<\lambda$, его след $F \cap X_{\iota}$ является окрестностью нуля. Это тоже верно, потому что топологии в $\left(X_{\lambda}\right)^{\star}$ и $\left(X_{\iota}\right)^{\star}$ задаются полярами одной и той же системы множеств в $X$ : во всех пространствах $\left(X_{\omega}\right)^{\star}$ базисные окрестности нуля - поляры множеств $S \in \mathscr{B} \mathscr{S}(X)$.

Итак, справедливо (3.14), и если заметить, что для всякого $\varkappa<\lambda$ это равенство естественно вписывается в диаграмму

(где $\sigma_{\varkappa}$ - вложение элемента индуктивной системы в соответствуюший индуктивный предел), то это будет означать вьполнение условия (iv) для инъективногоряда $\left(X_{\omega}\right)^{\star}$.

Мы убедились, что система $\left(X_{\omega}\right)^{\star}$ является инъективным рядом для пространства $X^{\star}$. По лемме 1.10 это означает, что между $\left(X_{\omega}\right)^{\star}$ и $\left(X^{\star}\right)_{\omega}$ имеется естественный изоморфизм с коммутативностью диаграммы (3.11).

Нам остается доказать утверждение (c). Это делается индукцией по индексу $\omega$ с помошью теоремы 3.10. Теорема доказана.

Аналогично доказывается

Теорема 3.13. Пусть $X-$ псевдонасыщенное ЛВП $и\left\{X^{\omega}, \sigma\right\}-$ инбективный ряд для X. Тогда:

(a) ингективный ряд $\left\{X^{\omega}\right\}$ состоит из псевдонасыщенных пространств;

(b) если $\left\{\left(X^{\star}\right)_{\omega}, \pi\right\}$ - проективный ряд для сопряженного пространства $X^{\star}$, то существует единственная система изоморфизмов

$$
\left(X^{\omega}\right)^{\star}=\left(X^{\star}\right) \omega
$$

такая, что для всякого $\varkappa<\lambda$ коммутативна диаграмма 
(с) для всякого морфизма псевдонасыщенных ЛВП $\varphi: X \rightarrow Y$ и любого ординала $\omega$ коммутативна диаграмма

в которой $Y^{\omega}-$ произвольный инвективный ряд для $Y, \quad\left(Y^{\star}\right)_{\omega}-$ произвольньи й проективный ряд для $Y^{\star}$, а $\varphi^{\omega} u\left(\varphi^{\star}\right)_{\omega}-$ системы морфизмов, определеннье в леммах 1.12 и 1.23 .

3.4. Двойственность между $\nabla$ и $\triangle$. Из теоремы 3.12 немедленно следует

Теорема 3.14. Пусть $X-$ псевдополное ЛВП. Тогда:

(а) существует единственньй изоморфизм $\left(X^{\Delta}\right)^{\star}=\left(X^{\star}\right)^{\nabla}$ такой, что коммутативна диаграмма

(b) для всякого морфизма псевдополных ЛВП $\varphi: X \rightarrow Y$ коммутативна диаграмма

Аналогично, из теоремы 3.13 следует

Теорема 3.15. Пусть $X-$ псевдонасыщенное ЛВП. Тогда:

(а) существует единственный изоморфизм $\left(X^{\nabla}\right)^{\star}=\left(X^{\star}\right)^{\Delta}$ такой, что коммутативна диаграмма 
(b) для всякого морфизма псевдонасыщенных ЛВП $\varphi: X \rightarrow Y$ коммутативна диаграмма

\section{§ 4. Стереотипные пространства}

4.1. Определения и примеры. ЛВП $X$ мы называем стереотипны.м, если оно одновременно псевдополно и псевдонасышено. Термин был введен автором в [8], и доводами в его пользу служили следующие соображения. Во-первых (как станет видно из примера 4.3), класс псевдополных псевдонасышенных пространств весьма широк (настолько, что включает все реально используемые в анализе локально выпуклые пространства). Во-вторых (как оказывается по результатам пп. 4.3 и 4.6), выйти из этого класса невозможно, если пользоваться стандартными (категорными) правилами конструирования новых пространств. Наконец, в-третьих, описанные в пп. 1.3 и 1.4 операции псевдопополнения и псевдонасышения позволяют строить для каждого локально выпуклого пространства ближайшие к нему в строго определенном смысле пространства из нашего класса, символизируюшие его стереотип (или cmepeomunb, поскольку остается пока неясным, коммутируют друг с другом операции псевдопополнения и псевдонасышения или нет).

Из теорем 2.14 и 2.16 следует

Теорема 4.1 (характеризация стереотипных пространств). Для ЛВП X следующие условия әквивалентны:

(i) пространство $X$ стереотипное;

(ii) отображсение $i_{X}: X \rightarrow X^{\star \star}$ является изоморфизмом.

Таким образом, класс стереотипных пространств выделяется равенством

$$
X^{\star \star}=X
$$

Впервые пространства с таким условием рефлексивности рассматривались в работе [4] в 1952 г. В ней было доказано, что равенству (4.1) удовлетворяют все банаховы и все рефлексивные в обычном смысле пространства. Затем сушественно позже, в 1967 г., Б. С. Брудовский показал, что похожее равенство эквивалентно выполнению неких условий, близких к тем, что мы называем псевдополнотой и псевдонасышенностью [5]. Параллельно им отмечалось, что равенству (4.1) удовлетворяют квазиполные бочечные пространства. Тот же факт независимо от него был установлен В. С. Уотерхаусом в работе [6] 1968 года. Наконец, независимо от всех, в 1973 г. К. Браунер в [7] отметил утверждение, в точности эквивалентное теореме 4.1.

Заметим, что из теоремы 4.1 (или 2.18) следует 
Теорема 4.2. Если $X$ - стереотипное пространство, то $X^{\star}$ - тоже стереотипное пространство.

Приведем некоторые примеры (из работ [5]-[7]).

ПРИмеР 4.3. Квазиполные бочечные пространства являются стереотипными в силу примера 1.13 .

ПримеР 4.4. Пространства Фреше и Браунера. Понятно, что всякое пространство Фреше $X$, как полное и бочечное, является стереотипным. Его сопряженное пространство $Y=X^{\star}$ также будет стереотипным по теореме 4.2. При этом если $\left\{U_{n}\right\}-$ 
счетная локальная база в $X$, то поляры $K_{n}=U_{n}^{\circ}$ являются компактами в $Y$, причем образуют фундаментальную систему компактов: каждый компакт $T \subseteq Y$ содержится в некотором компакте $K_{n}$ (отсюда следует, между прочим, что $Y$ не может быть пространством Фреше, если $X$ бесконечномерно). Пространства $Y$, сопряженные (в смысле определения $\S 2$ ) к пространствам Фреше $X$ впервые рассматривались К. Браунером в [24], и мы будем называть их пространствами Браунера. Характеристические свойства этих пространств полезно собрать в одном предложении.

Предложение 4.5. Для локально выпуклого пространства X следующие условия әквивалентнь:

(i) $Y$ является пространством Браунера;

(ii) $Y$ полно, является пространством Келли (т.е. всякое мнохсество $M \subseteq$ $Y$, оставляющее замкнутый след $M \cap K$ на любом компакте $K \subseteq Y$, замкнуто в $Y)$ и обладает счетной фундаментальной системой компактов $K_{n}: \forall T \in \mathscr{K}(Y)$ $\exists n \in \mathbb{N}: T \subseteq K_{n}$

(iii) $Y$ является стереотипным и обладает счетной фундаментальной системой компактов $K_{n}$;

(iv) $Y$ является стереотипньм и обладает счетной исчерпьвающей системой компактов $K_{n}: \bigcup_{n=1}^{\infty} K_{n}=Y$.

ПРИмеР 4.6. Пространства Банаха и Смит являются частными случаями пространств $\Phi$ реше и Браунера. Если $X$-пространство Банаха, то в силу $4.4 X$ и $Y=X^{\star}$ являются стереотипными пространствами. При этом шар $B$ в $Y$ имеет полярой так называемый универсальный компакт $K=B^{\circ}$ в $Y$, т.е. такой компакт, который поглощает любой другой компакт $T$ в $Y$. Пространства $Y=X^{\star}$, сопряженные в смысле определения $\S 2$ к пространствам Банаха $X$, первой рассматривала М. $\Phi$. Смит в [4], поэтому мы называем их пространствами Смит. Их характеристические свойства собраны в следующем предложении.

Предложение 4.7. Для локально выпуклого пространства Y следующие условия әквивалентны:

(i) $Y$ является пространством Смит;

(ii) $Y$ полно, является пространством Келли и обладает универсальным компактом $K: \forall T \in \mathscr{K}(X) \quad \exists \lambda \in \mathbb{C}: T \subseteq \lambda K$;

(iii) $Y$ является стереотипным пространством и обладает универсальным.м компактом;

(iv) $Y$ является стереотипным пространством и обладает компактной бочкой.

Пусть Ste обозначает класс стереотипных пространств, $\mathfrak{F} \mathfrak{e}$ - класс пространств $\Phi$ реше, $\mathfrak{B} \mathfrak{r a}$ - класс пространств Браунера, $\mathfrak{B a n}$ - класс пространств Банаха, $\mathfrak{S m i}-$ класс пространств Смит.

Связи меж ду этими классами иллюстрируются следуюшей диаграммой, в которой горизонтальные двойные стрелки означают переход к сопряженному пространству, а 
вертикальные связи - вложения:

Поскольку бесконечномерное пространство Смит не может быть бочечным, класс стереотипных пространств не ограничивается квазиполными бочечными. Более того, как оказывается, он содержит пространства, не являющиеся пространствами Макки.

ПРИМЕР 4.8 (стереотипное пространство, не являющееся пространством Макки). Таким пространством будет сопряженное $Y=X^{\star}$ к произвольному рефрлексивному (в обычном смысле) банахову пространству $X$. Действительно, в $X$ замкнутьй единичный шар $U$, как известно, будет $X^{\prime}$-слабо компактным, но не компактным в нормированной топологии множеством. Для сопряженного пространства $Y=X^{\star}$ это означает, что в нем имеется замкнутое выпуклое уравновешенное множество $K=U^{\circ}$, не являющееся окрестностью нуля, поляра которого $K^{\circ}=U$ является $Y$-слабым компактом в $Y^{\prime}$.

Приведем, наконец, примеры пространств, не являющихся стереотипными по разным причинам.

ПрИмер 4.9 (псевдополное, но не псевдонасыщенное пространство). Пусть $X-$ произвольное бесконечномерное банахово пространство, и пусть $Y=X_{\sigma}^{\prime}$ - его сопряженное пространство с $X$-слабой топологией. По теореме Банаха-Штейнгауза пространство $Y$ псевдополно. Покажем, что оно не стереотипное. Действительно, в $Y=$ $X_{\sigma}^{\prime}$ вполне ограниченные множества - то же самое, что ограниченные, и то же самое, что подмножества в полярах $U^{\circ}$ окрестностей нуля $U$ из $X$. Поэтому $Y^{\star}=X$, а $Y^{\star \star}=X^{\star} \neq X_{\sigma}^{\prime}=Y$.

ПРИМеР 4.10 (псевдонасъщенное, но не псевдополное пространство). Таким пространством в силу примера 1.14 будет произвольное неполное метризуемое пространство.

4.2. Двойственность в классе стереотипных пространств. В силу теоремы 4.1 каждое стереотипное пространство $X$ однозначно восстанавливается по своему сопряженному пространству $Y=X^{\star}$. Поэтому различным свойствам $X$ должны соответствовать их двойственные аналоги в $Y$ и наоборот. Если собрать в одном предложении наиболее очевидные закономерности такого рода, возникает следующая любопытная картина.

Теорема 4.11. Пусть $\langle X, Y\rangle$ - дуальная система стереотипных пространств, m.e. $X^{\star}=Y$ u $Y^{\star}=X$. Тогда:

(а) $X$ нормируемо $\Longleftrightarrow X-$ пространство Банаха $\Longleftrightarrow Y-$ пространство Cмит; 
(b) $X$ метризуемо $\Longleftrightarrow X-$ пространство фреше $\Longleftrightarrow Y-$ пространство Браунера;

(c) $X$ бочечно $\Longleftrightarrow Y$ обладает свойством Гейне-Бореля;

(d) $X$ квазибочечно $е$ в $Y$ любое подмножество $T$, поглощаемое любой бочкой, вполне ограничено;

(е) $X$ - пространство Макки $е$ в всякое $Y^{\prime}$-слабо компактное множество компактно;

(f) $X$ - монтелевское пространство $\Longleftrightarrow Y$-монтелевское пространство;

(g) $X$ - пространство со слабой топологией $е$ в номерен;

(h) X сепарабельно (т.е. обладает счетным всюду плотным мнохсеством) $\Longleftrightarrow$ в Y существует последовательность замкнутых подпространств $L_{n}$ конечной коразмерности $\left(\forall n \operatorname{dim}\left(Y / L_{n}\right)<\infty\right)$ с тривиальным пересечением: $\bigcap_{n=1}^{\infty} L_{n}=$ $\{0\}$.

(i) $X$ обладает (классическим) свойством аппроксимачии $\Longleftrightarrow$ обладает (классическим) свойством аппроксимачии;

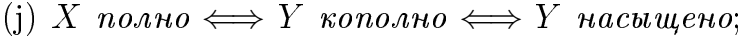

(k) $X$ - пространство Птака (совершенно полное в смысле [15]) всякое подпространство $L$, оставляющее замкнутый след $L \cap K$ на каждом компакте $K \subseteq Y$, автоматически замкнуто;

(1) $X$ - әиперполное пространство (в смысле [15]) $в Y$ всякое выпуклое уравновешенное множсество $B$, оставляющее замкнутый след $B \cap K$ на каждом компакте $K \subseteq Y$, автоматически замкнуто.

Для доказательства достаточно заметить, что операция перехода к поляре $B \mapsto B^{\circ}$ устанавливает биекцию между системами замкнутых выпуклых уравновешенных множеств в $X$ и $Y$, причем окрестность нуля переходит в компакт, а бочка - в ограниченное множество.

4.3. Лемма об аннуляторе. Следующая лемма будет играть важную роль в дальнейших построениях.

Лемма 4.12. Пусть $X$ - стереотипное пространство, $E$ - его замкнутое локально выпуклое подпространство, и пусть

$$
E^{\perp}=\left\{f \in X^{\star}:\left.f\right|_{E}=0\right\}
$$

- аннулятор пространства $E$ в сопряженном пространстве $X^{\star}$. Тогда:

(а) имеется естественный изоморфизм локально выпукльх пространств

$$
E^{\star} \cong X^{\star} / E^{\perp}
$$

порождающий изоморфизмы стереотипных пространств

$$
\left(E^{\Delta}\right)^{\star} \cong\left(X^{\star} / E^{\perp}\right)^{\nabla}, \quad E^{\Delta} \cong\left[\left(X^{\star} / E^{\perp}\right)^{\nabla}\right]^{\star} ;
$$

(b) имеется естественный изоморфизм локально выпуклых пространств

$$
\left(E^{\perp}\right)^{\star} \cong X / E,
$$

порождающий изоморфизмы стереотипных пространств

$$
\left(\left(E^{\perp}\right)^{\Delta}\right)^{\star} \cong(X / E)^{\nabla}, \quad\left(E^{\perp}\right)^{\Delta} \cong\left[(X / E)^{\nabla}\right]^{\star} .
$$


ДокАЗАТЕЛЬСТво. Каждому функционалу $g \in E^{\star}$ поставим в соответствие множество $\tilde{g} \subseteq X^{\star}$ всевозможных его линейных непрерывных продолжений на $X$ :

$$
\tilde{g}=\left\{f \in X^{\star}:\left.f\right|_{E}=g\right\} .
$$

Ясно, что $\tilde{g}$ является классом эквивалентности относительно $E^{\perp}$, т.е. $\tilde{g} \in X^{\star} / E^{\perp}$, причем отображение $g \in E^{\star} \mapsto \tilde{g} \in X^{\star} / E^{\perp}$ биективно. Докажем его непрерывность в обе стороны.

1) Пусть $V \in \mathscr{B} \mathscr{U}\left(X^{\star} / E^{\perp}\right)$; тогда $V=\pi(U)$, где $\pi: X^{\star} \rightarrow X^{\star} / E^{\perp}-$ факторотображение, а $U \in \mathscr{B} \mathscr{U}\left(X^{\star}\right), U+E^{\perp}=U$. Поскольку $X$ стереотипное, то $U=K^{\circ} X^{\star}$, где $K \in \mathscr{B} \mathscr{K}(X)$. Условие $K^{\circ}+E^{\perp}=K^{\circ}$ означает, что $K \subseteq E$. Поэтому окрестность нуля $W=K_{E^{\star}}^{\circ} \in \mathscr{B} \mathscr{U}\left(E^{\star}\right)$ будет прообразом $V$ при отображении $g \in E^{\star} \mapsto \tilde{g} \in$ $X^{\star} / E^{\perp}$.

2) Наоборот, если имеется базисная окрестность нуля $W=K_{E^{\star}}^{\circ} \in \mathscr{B} \mathscr{U}\left(E^{\star}\right), K \in$ $\mathscr{B} \mathscr{K}(E)$, то можно рассмотреть $U=K_{X^{\star}}^{\circ} \in \mathscr{B} \mathscr{U}\left(X^{\star}\right)$ и положить $V=\pi(U)$, и мы получим $\widetilde{W}=V$.

Мы доказали формулу (4.3). Из нее следует

$$
\left(E^{\star}\right)^{\nabla} \cong\left(X^{\star} / E^{\perp}\right)^{\nabla} \text {. }
$$

Теперь, воспользовавшись теоремой 3.14 , мы получим

$$
\left(E^{\Delta}\right)^{\star} \cong\left(E^{\star}\right)^{\nabla} \cong\left(X^{\star} / E^{\perp}\right)^{\nabla},
$$

и уже отсюда

$$
E^{\Delta} \cong\left(E^{\Delta}\right)^{\star \star} \cong\left[\left(X^{\star} / E^{\perp}\right)^{\nabla}\right]^{\star} .
$$

Таким образом, доказано (a). Переобозначив объекты, мы получим условие (b). Лемма доказана.

4.4. Непосредственное подпространство и непосредственное факторпространство. Класс $\mathfrak{S t e ~ с т е р е о т и п н ы х ~ п р о с т р а н с т в ~ о б р а з у е т ~ к а т е г о р и ю , ~ м о р ф и з м а м и ~}$ в которой служат линейные непрерывные отображения $\varphi: X \rightarrow Y$. Легко проверить, что в этой категории, как и в категории $\mathfrak{L} \mathfrak{C}$, мономорфизмами будут инъективные отображения (т.е. такие, что $\left.\varphi^{-1}(0)=\{x \in X: \varphi(x)=0\}=\{0\}\right)$, а эпиморфизмамиотображения с плотным образом (т.е. такие, что $\overline{\varphi(X)}=\overline{\{\varphi(x) ; x \in X\}}=Y$ ). Напомним, что морфизм называется биморфизмом, если он одновременно мономорфизм и эпиморфизм.

Стереотипное пространство $Y$ мы называем подпространством стереотипного пространства $X$, если задан (произвольный) мономор физм стереотипных пространств $\mu: Y \rightarrow X$, называемый представляющим мономорфизмом подпространства $Y$ в пространстве $X$.

Если $Y$ и $\widetilde{Y}$ - два подпространства в стереотипном пространстве $X$ с представляюшими мономорфизмами $\mu$ и $\tilde{\mu}$, причем имеется биморфизм $\beta: Y \rightarrow \tilde{Y}$, замыкаюший диаграмму 
то подпространство $\widetilde{Y}$ назьвается посредником подпространства $Y$ в пространстве $X$.

Подпространство $Y$ стереотипного пространства $X$ мы называем непосредственныц. подпространством в $X$, если у него нет посредников, т.е. если для любого его посредника $\widetilde{Y}$ в $X$ соответствуюший биморфизм $\beta$ в диаграмме (4.7) автоматически является изоморфизмом.

ПримеР 4.13. Пусть $X$ - стереотипное пространство и $E$ - его замкнутое локально выпуклое подпространство (т.е. подпространство в алгебраическом смысле, являюшееся одновременно замкнутым подмножеством топологического пространства $X$ ). Тогда ЛВП $E$ (с индуцированной из $X$ топологией) будет псевдополным, поэтому его псевдонасышение $E^{\Delta}$ будет также псевдополным в силу таблишы инвариантов п. 1.5. Значит, $E^{\Delta}$ является стереотипным пространством. Ясно, что оно будет непосредственным подпространством стереотипного пространства $X$ с представляющим мономорфизмом $\sigma^{\Delta}: E^{\Delta} \rightarrow X$, где $\sigma: E \subseteq X$ - естественное вложение (локально выпуклых пространств).

Следуюшая теорема утверждает, что эта конструкция описывает все непосредственные подпространства.

Теорема 4.14 (о строении непосредственных подпространств). Стереотипное пространство $Y$ тогда и только тогда будет непосредственньц подпространством стереотипного пространства $X$ с представляющим мономорфизмом $\mu: Y \rightarrow$ $X$, когда найдутся:

- замкнутое подпространство $E$ локально выпуклого пространства $X$,

- изоморфизм стереотипных пространств $\beta: Y \rightarrow E^{\Delta}$, такие, что коммутативна диаграмма

в которой $\sigma^{\Delta}$ - псевдонасыщение естественного вложения $\sigma: E \subseteq X$.

Для доказательства в качестве $E$ нужно взять $\overline{\mu(Y)}$.

Стереотипное пространство $Y$ мы называем факторпространством стереотипного пространства $X$, если задан (произвольный) эпиморфизм стереотипных пространств $\varepsilon: X \rightarrow Y$, называемый представляющим әпиморфизмом факторпространства $Y$ пространства $X$.

Если $Y$ и $\widetilde{Y}$ - два факторпространства стереотипного пространства $X$ с представляюшими эпиморфизмами $\varepsilon$ и $\tilde{\varepsilon}$, причем имеется биморфизм $\beta: \widetilde{Y} \rightarrow Y$, замыкаюший диаграмму 
то факторпространство $\widetilde{Y}$ назьвается посредником факторпространства $Y$ пространства $X$.

Факторпространство $Y$ стереотипного пространства $X$ мы называем непосредственным факторпространством пространства $X$, если у него нет посредников, т.е. если для любого его посредника $\widetilde{Y}$ соответствуюший биморфизм $\beta$ в диаграмме (4.9) автоматически является изоморфизмом.

ПримеР 4.15. Пусть $X$ - стереотипное пространство и $E$ - его замкнутое локально выпуклое подпространство (т.е. подпространство в алгебраическом смысле, являюшееся одновременно замкнутым подмножеством топологического пространства $X$ ). Тогда факторпространство локально выпуклых пространств $X / E$ будет в силу таблишы инвариантов п. 1.5 псевдонасышенным локально выпуклым пространством. Значит, опять же в силу таблицы инвариантов, его псевдопополнение $(X / E)^{\nabla}$ будет псевдонасыщенным (и псевдополным), т.е. стереотипным пространством. Покажем, что оно будет непосредственным факторпространством пространства $X$ с представляюшим эпиморфизмом $\pi^{\nabla}: X \rightarrow(X / E)^{\nabla}$, где $\pi: X \rightarrow X / E$ - факторотображение локально выпуклых пространств. Действительно, аннулятор $E^{\perp}-$ замкнутое локально вьпуклое подпространство в $X^{\star}$. По лемме 4.12 , (b) факторпространство $(X / E)^{\nabla}$ стереотипного пространства $X$ имеет сопряженным пространством $\left[(X / E)^{\nabla}\right]^{\star}$ подпространство $\left(E^{\perp}\right)^{\Delta}$ стереотипного пространства $X^{\star}$. Поскольку в силу примера $4.13\left(E^{\perp}\right)^{\Delta}-$ непосредственное подпространство в $X^{\star}$, по принципу двойственности получаем, что $(X / E)^{\nabla}$ должно быть непосредственным факторпространством для $X$.

Применяя теперь принцип двойственности к теореме 4.14, мы получим утверждение об универсальности конструкции примера 4.15 .

Теорема 4.16 (о строении непосредственных факторпространств). Стереотипное пространство Y тогда и только тогда будет непосредственным факторпространством стереотипного пространства $X$ с представляющим эпиморфизмом $\varepsilon: X \rightarrow Y$, когда найдутся:

- замкнутое подпространство Е локально выпуклого пространства $X$,

- изоморфизм стереотипных пространств $\beta:(X / E)^{\nabla} \rightarrow Y$, такие, что коммутативна диаграмма

в которой $\pi^{\nabla}$ - псевдопополнение факторотображсения $\pi: X \rightarrow X / E$.

4.5. Предабелевость категории $\mathfrak{S t e . ~ П о с к о л ь к у ~ л ю б ы е ~ д в а ~ п а р а л л е л ь н ы х ~ м о р - ~}$ физма $\varphi, \psi: X \rightarrow Y$ в категории стереотипных пространств $\mathfrak{S t e}_{\mathfrak{t}}$ можно складывать и вычитать, понятно, что $\mathfrak{S t e ~ я в л я е т с я ~ а д д и т и в н о и ̆ ~ к а т е г о р и е и ̆ . ~ Н е ~ т а к ~ о ч е в и д н о , ~ ч т о ~ о н а ~}$ предабелева. 
Теорема 4.17. Всякий морфизм стереотипных пространств $\varphi: X \rightarrow Y$ обладаem:

- ядром $\operatorname{ker} \varphi: \operatorname{Ker} \varphi \rightarrow X$,

- коядром $\operatorname{coker} \varphi: Y \rightarrow \operatorname{Coker} \varphi$,

- образом $\operatorname{im} \varphi: \operatorname{Im} \varphi \rightarrow Y$

- кообразом $\operatorname{coim} \varphi: X \rightarrow \operatorname{Coim} \varphi$

в категории Ste стереотипных пространств. При этом операция $\varphi \mapsto \varphi^{\star}$ перехода к сопряженному отображению устанавливает следующие связи между этими обвектами:

$$
\begin{array}{cl}
(\operatorname{ker} \varphi)^{\star}=\operatorname{coker} \varphi^{\star}, & (\operatorname{coker} \varphi)^{\star}=\operatorname{ker} \varphi^{\star}, \\
(\operatorname{im} \varphi)^{\star}=\operatorname{coim} \varphi^{\star}, & (\operatorname{coim} \varphi)^{\star}=\operatorname{im} \varphi^{\star}, \\
(\operatorname{Ker} \varphi)^{\perp \Delta}=\operatorname{Im} \varphi^{\star}, & (\operatorname{Im} \varphi)^{\perp \Delta}=\operatorname{Ker} \varphi^{\star}, \\
\operatorname{Ker} \varphi=\left(\operatorname{Im} \varphi^{\star}\right)^{\perp \Delta}, & \operatorname{Im} \varphi=\left(\operatorname{Ker} \varphi^{\star}\right)^{\perp \Delta} .
\end{array}
$$

ДокАЗАТЕЛЬСТво. Прообраз нуля $\varphi^{-1}(0)$ будет, очевидно, замкнутым локально выпуклым подпространством в $X$. Поэтому его псевдонасышение $\left[\varphi^{-1}(0)\right]^{\Delta}$ будет в силу примера 4.13 (непосредственным) подпространством стереотипного пространства $X$. Положив $\operatorname{Ker} \varphi=\left[\varphi^{-1}(0)\right]^{\Delta}$, мы получим, что всякий морфизм $\psi: Z \rightarrow X$ со свойством $\varphi \circ \psi=0$ автоматически поднимается до морфизма локально выпуклых пространств $\tilde{\psi}: Z \rightarrow \varphi^{-1}(0)$ и, поскольку $Z$ псевдонасышено, до морфизма $\tilde{\psi}: Z \rightarrow$ $\left[\varphi^{-1}(0)\right]^{\Delta}=\operatorname{Ker} \varphi$ (здесь применяется диаграмма (1.21)). Таким образом, $\operatorname{Ker} \varphi$ действительно является ядром морфизма $\varphi$ в категории Ste.

Положив Coker $\varphi=[Y / \overline{\varphi(X)}]^{\nabla}$, получим, что из леммы 4.12 следует равенство Coker $\varphi=\left[\operatorname{Ker} \varphi^{\star}\right]^{\star}$, которое в силу принципа двойственности должно означать, что Coker $\varphi$ действительно является коядром для $\varphi$. Сушествование ядра и коядра автоматически влечет за собой существование образа и кообраза, которые в данном случае принимают вид $\operatorname{Im} \varphi=[\overline{\varphi(X)}]^{\Delta}$ и $\operatorname{Coim} \varphi=\left[X / \varphi^{-1}(0)\right]^{\nabla}$.

Формулы (4.11) следуют из принципа двойственности и влекут за собой (4.12). Например, с помошью (4.6) получаем

$$
\operatorname{Ker} \varphi=\left(\operatorname{Coker} \varphi^{\star}\right)^{\star}=\left(\left(X^{\star} / \operatorname{Im} \varphi^{\star}\right)^{\nabla}\right)^{\star} \stackrel{(4.6)}{=}\left(\operatorname{Im} \varphi^{\star}\right)^{\perp \Delta} .
$$

Теорема доказана.

Из предабелевости категории Ste следует, что всякий морфизм $\varphi: X \rightarrow Y$ в Ste раскладывается в композицию

в которой морфизм $\operatorname{bim} \varphi$ называется биморфизмом, ассоциированным $с \varphi$. 
4.6. Иммерсии и субмерсии. Условимся говорить, что образ морфизма $\mu: X \rightarrow$ $Y$ шире образа морфизма $\varphi: Z \rightarrow Y$ :

$$
\operatorname{Im} \varphi \leqslant \operatorname{Im} \mu,
$$

если для всякого морфизма $\lambda: Y \rightarrow L$ из равенства $\lambda \circ \mu=0$ следует равенство $\lambda \circ \varphi=0$.

Морфизм стереотипных пространств $\mu: X \rightarrow Y$ называется иммерсией, если для всякого морфизма $\varphi: Z \rightarrow Y$ такого, что $\operatorname{Im} \varphi \leqslant \operatorname{Im} \mu$, найдется единственный морфизм $\psi: Z \rightarrow X$, замыкающий диаграмму

Теорема 4.18. Для морфизма стереотипных пространств $\mu: X \rightarrow Y$ следующие условия эквивалентны:

(i) $\mu$ является иммерсией;

(ii) $\mu$ совпадает со своим образом: $\mu=\operatorname{im} \mu$ (m.e. $\operatorname{coim} \mu u \operatorname{bim} \mu$ являются изоморфизмами);

(iii) $X$ является непосредственным подпространством в Y с представляющим мономорфизмом $\mu$.

Рассмотрим двойственную конструкцию. Условимся говорить, что ядро морфизма $\varepsilon: X \rightarrow Y$ уже ядра морфизма $\varphi: Z \rightarrow Y$, т.е. $\operatorname{Ker} \varepsilon \leqslant \operatorname{Ker} \varphi$, если для всякого морфизма $\lambda: L \rightarrow X$ из равенства $\varepsilon \circ \lambda=0$ следует равенство $\varphi \circ \lambda=0$.

Морфизм стереотипных пространств $\varepsilon: X \rightarrow Y$ называется субмерсией, если для всякого морфизма $\varphi: X \rightarrow Z$ такого, что

$$
\operatorname{Ker} \varepsilon \leqslant \operatorname{Ker} \varphi
$$

найдется единственный морфизм $\psi: Y \rightarrow Z$, замыкающий диаграмму

Теорема 4.19. Для морфизма стереотипных пространств $\varepsilon: X \rightarrow Y$ следующие условия әквивалентны:

(i) в является субмерсией;

(ii) $\varepsilon$ совпадает со своим кообразом: $\varepsilon=\operatorname{coim} \varepsilon$ (m.е. im $\varepsilon$ u bim $\varepsilon$ являются изоморфизмами);

(iii) $Y$ является непосредственным факторпространством для $X$ с представляющим эпиморфизмом $\varepsilon$.

4.7. Полнота категории Ste. Из таблищы инвариантов п. 1.5 следует 
Теорема 4.20. Всякое семейство $\left\{X_{i} ; i \in I\right\}$ стереотипных пространств обладает прямой суммой и прямым произведением в категории стереотипных пространств $\mathfrak{S t e . ~}$

(Эти объекты будут совпадать с обычной локально выпуклой прямой суммой и прямым произведением.)

Теорема 4.21. Всякая индуктивная (проективная) система $\left\{X_{i} ; i \in I\right.$, $i \rightarrow \infty\}$ стереотипных пространств обладает индуктивным (проективным) пределом в категории стереотипных пространств Ste.

С обычным локально выпуклым индуктивным и проективным пределом эти объекты связаны формулами

$$
\mathfrak{S t e -} \lim _{i \rightarrow \infty} X_{i}=\left(\mathfrak{L C S}-\lim _{i \rightarrow \infty} X_{i}\right)^{\nabla}, \quad \mathfrak{S t e}-\underbrace{\lim }_{i \rightarrow \infty} X_{i}=(\mathfrak{L C S}-\underbrace{\lim _{i \rightarrow \infty}}_{i \rightarrow \infty} X_{i})^{\Delta} .
$$

\section{Список литературы}

1. Pontrjagin L.S. The theory of topological commutative croups // Ann. Math. 1934. V. 35. № 2. P. 361-388.

2. Van Kampen E. Locally bicompact Abelian groups and their character groups // Ann. Math. 1935. V. 36. № 2. P. $448-456$.

3. Venkataraman R. Characterization of Pontryagin duality // Math. Z. 1976. V. 149. № 2. P. 109-119.

4. Smith M. F. The Pontrjagin duality theorem in linear spaces // Annals of Mathematics. 1952. V. 56. № 2. P. $248-253$.

5. Брудовский Б. С. О $k$-и с-рефлексивности // Литовский матем. сб. 1967. Т. 7. № 1. С. 17-21.

6. Waterhouse W. C. Dual groups of vector spaces // Pacific J. Math. 1968. V. 26. № 1. P. 193-196.

7. Brauner K. Duals of Frechet spaces and a generalization of the Banach-Dieudonne theorem // Duke Math. Jour. 1973. V. 40. № 4. P. 845-855.

8. Акбаров С. С. Двойственность Понтрягина в теории топологических векторных пространств // Матем. заметки. 1995. Т. 57. № 3. С. 463-466.

9. Акбаров С. С. Двойственность Понтрягина в теории топологических модулей. // Функц. анализ и его прилож. 1995. Т. 29. № 4. С. 68-72.

10. Акбаров C.C. Свойство стереотипной аппроксимации и проблема Гротендика однозначности следа // Функц. анализ и его прилож. 1999. Т. 33. № 2. С. 68-73.

11. Шефер X. Топологические векторные пространства. М.: Мир, 1971.

12. Энгелькинг Р. Общая топология. М.: Мир, 1986.

13. Келли Дж. Л. Общая топология. М.: Наука, 1981.

14. Смолянов О.Г. Пространство $\mathscr{D}(X)$ не является наследственно полным // Изв. АН CССР. Сер. матем. 1971. Т. 35. №4. С. 686-696.

15. Робертсон А. П., Робертсон В. Дж. Топологические векторные пространства. М.: Мир, 1967. 